Comment. Math. Helv. 75 (2000) 701-736

(C) 2000 Birkhäuser Verlag, Basel

0010-2571/00/040701-36\$1.50+0.20/0

Commentarii Mathematici Helvetici

\title{
Rigidity of quasi-isometries for some hyperbolic buildings
}

Marc Bourdon and Hervé Pajot

\begin{abstract}
For a family of negatively-curved buildings, we show that any quasi-isometry lies within bounded distance from an isometry.
\end{abstract}

Mathematics Subject Classification (2000). Primary: 30C65, 51E24.

Keywords. Hyperbolic building, quasi-conformal mapping, conformal modulus, quasi-isometry, rigidity.

\section{Introduction}

\subsection{Statement of the main result}

A map $F: X \longrightarrow Y$ between metric spaces is a quasi-isometry if there exist constants $C \geq 1$ and $D \geq 0$ such that

$$
C^{-1} d_{X}\left(x, x^{\prime}\right)-D \leq d_{Y}\left(F(x), F\left(x^{\prime}\right)\right) \leq C d_{X}\left(x, x^{\prime}\right)+D
$$

whenever $x$ and $x^{\prime}$ are in $X$, and if $d_{Y}(y, f(X)) \leq D$ for every $y \in Y$.

In this paper, we study quasi-isometries on a class of Tits buildings that we call right-angled Fuchsian buildings: their apartments are hyperbolic planes and their chambers are regular hyperbolic $p$-gons with right angles (see part 1 below for more details). These buildings admit a length metric whose curvature is less than or equal to -1 . The main result of the paper is

Theorem. Let $\Delta$ and $\Delta^{\prime}$ be right-angled Fuchsian buildings. Then, any quasiisometry $F: \Delta \longrightarrow \Delta^{\prime}$ lies within bounded distance from an isometry.

The analogous statement was proved by P. Pansu (see [Pa]) for quaternionic and Cayley hyperbolic spaces, by developing a geometric analysis for CarnotCaratheodory spaces. Using M. Gromov's notion of asymptotic cone, B. Kleiner

The second author is partially supported by the European Commission (TMR 1998-2001 Network Harmonic Analysis). 
and B. Leeb have proved this statement for irreducible affine buildings and irreducible symmetric spaces of higher rank (see [KL1], [KL2]; see also [EF] for another proof). M. Kapovich, B. Kleiner, B. Leeb and R. Schwartz have established it for universal covers of compact manifolds with curvature -1 , of dimension at least 3 and with a non empty totally geodesic boundary. Their proof (not written) is based on a doubling construction and on the technique of R. Schwartz's paper [Sc]. Recently, M. Kapovich and B. Kleiner [KK] have produced examples of word hyperbolic groups where rigidity of quasi-isometries holds for purely topological reasons. As an immediate consequence of our main result, we have

Corollary. a) Let $G$ be a group of finite type. Suppose that $G$ is quasi-isometric (relatively to a word metric) to a right-angled Fuchsian building $\Delta$. Then, $G$ admits the following decomposition

$$
1 \rightarrow N \rightarrow G \rightarrow \Gamma \rightarrow 1
$$

where $N$ is a finite normal subgroup of $G$ and $\Gamma$ is a co-compact lattice of the group of isometries of $\Delta$.

b) (Mostow-type rigidity) Let $\Delta$ and $\Delta^{\prime}$ be two right-angled Fuchsian buildings and let $\Gamma$ and $\Gamma^{\prime}$ be two co-compact lattices of the group of isometries of $\Delta$ and $\Delta^{\prime}$ respectively. Then, any isomorphism from $\Gamma$ to $\Gamma^{\prime}$ is conjugation by an isometry from $\Delta$ to $\Delta^{\prime}$.

Note that the Mostow rigidity for right-angled Fuchsian buildings is established more directly in [B1].

Remarks and questions. a) Examples of lattices of the group of isometries of hyperbolic buildings are studied in $[\mathrm{H}],[\mathrm{HP}],[\mathrm{GP}],[\mathrm{L}],[\mathrm{R}],[\mathrm{B} 1]$, [B2], [B3]. However, a little is known on these groups. For instance, we do not know if co-compact lattices of the same hyperbolic building are all commensurable.

b) It seems possible that our main theorem remains true for some other Fuchsian buildings, and maybe for all of them (see [B2] for definitions). However, some arguments of this paper use the notion of «tree-wall» which is particular to buildings with right angles (see section 1.2). In section 4.3, we will collect results of this paper which remain true in the case of more general buildings.

\subsection{Sketch of proof}

The strategy of the proof is inspired by that of Pansu for quaternionic and Cayley hyperbolic spaces (see $[\mathrm{Pa}]$ ). The main idea is to study the quasi-conformal structure of the boundary at infinity of hyperbolic buildings $\Delta$.

Recall that a homeomorphism $f: X \rightarrow Y$ (where $X$ and $Y$ are metric spaces) is quasi-symmetric if there exists a homeomorphism $\phi:[0,+\infty) \rightarrow[0,+\infty)$ so that

$$
d_{X}(x, a) \leq t d_{X}(x, b) \Rightarrow d_{Y}(f(x), f(a)) \leq \phi(t) d_{Y}(f(x), f(b))
$$


whenever $x, a, b$ are in $X$, and $t$ in $[0,+\infty)$.

Note that the inverse of a quasi-symmetric homeomorphism and the composition of two quasi-symmetric homeomorphisms are quasi-symmetric as well.

If $f: X \rightarrow Y$ is a homeomorphism, we define for every $x \in X$ and $r>0$,

$$
\begin{aligned}
& L_{f}(x, r)= \sup \left\{d_{Y}\left(f(x), f\left(x^{\prime}\right)\right): d_{X}\left(x, x^{\prime}\right) \leq r\right\}, \\
& l_{f}(x, r)= \inf \left\{d_{Y}\left(f(x), f\left(x^{\prime}\right)\right): d_{X}\left(x, x^{\prime}\right) \geq r\right\}, \\
& L_{f}(x)=\limsup _{r \rightarrow 0} \frac{L_{f}(x, r)}{r}, \\
& \text { and } l_{f}(x)=\liminf _{r \rightarrow 0} \frac{l_{f}(x, r)}{r} .
\end{aligned}
$$

Assume that $X$ and $Y$ have finite Hausdorff dimensions. Denote by $H_{X}$ and $H_{Y}$ their Hausdorff dimensions and by $\mathcal{H}_{X}$ and $\mathcal{H}_{Y}$ their Hausdorff measures (see [Mat] for definitions). We say that $f$ is conformal if $f$ is quasi-symmetric and satisfies

(i) $L_{f}(x)=l_{f}(x) \in(0,+\infty)$ for $\mathcal{H}_{X}$-almost every $x \in X$;

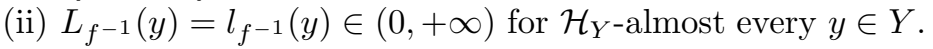

The main steps of the proof are the following. Let $\Delta$ be a right-angled Fuchsian building. Its boundary at infinity (that we denote by $\partial \Delta$ ) carries a canonical metric called combinatorial metric, which is induced by the building structure (see section 2.1). An abstract theorem of M. Gromov [G] implies that any quasiisometry $F: \Delta \rightarrow \Delta^{\prime}$ induces a quasi-symmetric homeomorphism $f: \partial \Delta \rightarrow \partial \Delta^{\prime}$. By a fine analysis of the metric structure of the boundary, we show that

$-f$ is a conformal homeomorphism from $\partial \Delta$ to $\partial \Delta^{\prime}$ (theorem 3.0); $4.0)$.

- $f$ is the extension to the boundaries of an isometry from $\Delta$ to $\Delta^{\prime}$ (theorem

Our theorem follows from these results.

Remarks. a) The definition of «quasi-symmetric homeomorphisms» differs from authors. Our definition is due to P. Tukia and J. Väisälä [TV]. In [HK], J. Heinonen and P. Koskela use the following definition which seems a priori weaker.

A homeomorphism $f: X \rightarrow Y$ is (weakly) quasi-symmetric if there exists a constant $C>1$ so that

$$
\frac{L_{f}(x, r)}{l_{f}(x, r)} \leq C
$$

whenever $x \in X$ and $r>0$. By a theorem of J. Väisäliä [V1], these two definitions are equivalent if $X$ and $Y$ are path-wise connected and carry a doubling measure.

b) Recall that a homeomorphism $f: X \rightarrow Y$ is quasi-conformal if there exists a constant $C>0$ so that

$$
\limsup _{r \rightarrow 0} \frac{L_{f}(x, r)}{l_{f}(x, r)} \leq C
$$


whenever $x \in X$. If $X$ and $Y$ are bounded, Ahlfors-regular (with the same dimension bigger than 1) Loewner spaces (see section 2), a quasi-conformal homeomorphism is quasi-symmetric by a theorem of J. Heinonen and P. Koskela (see [HK] theorem 4.9). In particular, this result applies to the boundary of right-angled Fuchsian buildings.

\subsection{Organization of the paper}

In part 1 we define the buildings $\Delta$ and we study shortly their building structure. Part 2 is devoted to the careful study of the boundary of $\Delta$. Its combinatorial metrics and its conformal structure are discussed in section 2.1. We study curves and geodesics of $\partial \Delta$ in section 2.2 and the Loewner structure of $\partial \Delta$ in section 2.3. In part 3, we show that quasi-symmetric homeomorphisms $f: \partial \Delta \rightarrow \partial \Delta^{\prime}$ are conformal. In part 4 , we prove that conformal homeomorphisms $f: \partial \Delta \rightarrow \partial \Delta^{\prime}$ are extensions to the boundaries of isometries from $\Delta$ onto $\Delta^{\prime}$.

\section{Right-angled Fuchsian buildings}

In this paper, we deal with buildings that we call right-angled Fuchsian buildings. Throughout the paper, they will be denoted by $\Delta$. In section 1.1 , we recall their definition following [B2] section 1.5.1. We also discuss their negatively curved geometry and the existence of lattices in their group of isometries. Section 1.2 is devoted to the description of their building structure and to the definition of the notion of trees-walls that will be a useful tool in part 2 .

\subsection{Definition and basic properties of $\Delta$}

Let $r$ be an integer with $r \geq 5$, let $R$ be a regular $r$-gon with right angles in the hyperbolic plane $H_{\mathbf{R}}^{2}$ with curvature -1 , and let $\left(q_{1}, \ldots, q_{r}\right)$ be a $r$-tuple of integers so that $q_{i} \geq 2$ for $i=1, . ., r$.

We label clockwise the edges of $R$ by $\{1\}, \ldots,\{r\}$ and its vertices by $\{1,2\}, \ldots$, $\{r-1, r\},\{r, 1\}$ (see figure 1 for $r=5$ ). Then, we define an orbihedron structure on $R$ as follows. To the face of $R$, we attach the trivial group; to the edge $\{i\}$, the group $\Gamma_{i}=\mathbf{Z} /\left(q_{i}+1\right) \mathbf{Z}$; to the vertex $\{i, i+1\}$, the group $\Gamma_{i, i+1}=\Gamma_{i} \times \Gamma_{i+1}$. By an abstract theorem of A. Haefliger ([Hae]), this orbihedron is developable; its universal cover, denoted by $\Delta$, is a labeled cell 2-complex with the following properties:

(i) $\Delta$ is contractible;

(ii) its edges and its vertices are labeled by the same symbols as above;

(iii) its 2-cells are copies of the labeled complex $R$;

(iv) each edge labeled by $\{i\}$ belongs to $\left(q_{i}+1\right) 2$-cells, the link of each vertex labeled by $\{i, i+1\}$ is the complete bipartite graph with $\left(q_{i}+1\right)+\left(q_{i+1}+1\right)$ vertices (see figure 2 in the case $\left(q_{i}, q_{i+1}\right)=(2,3)$ ). 


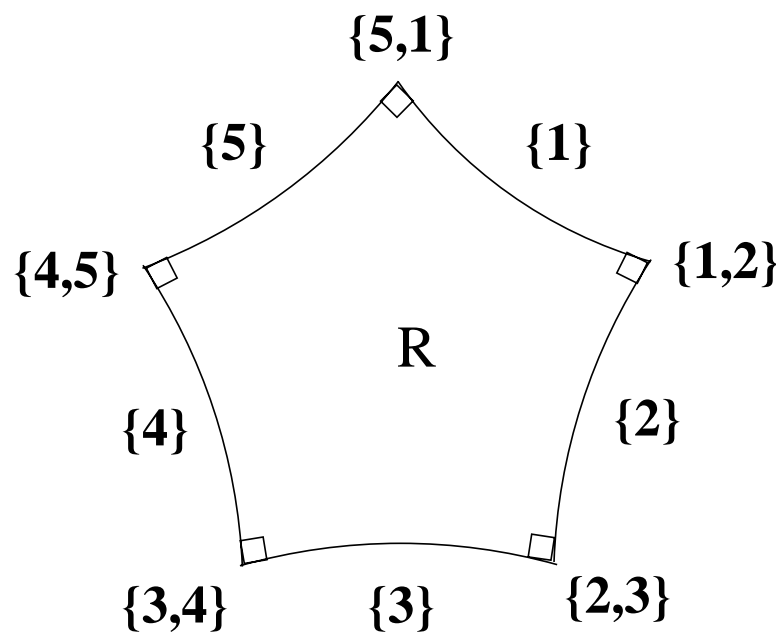

Figure 1.

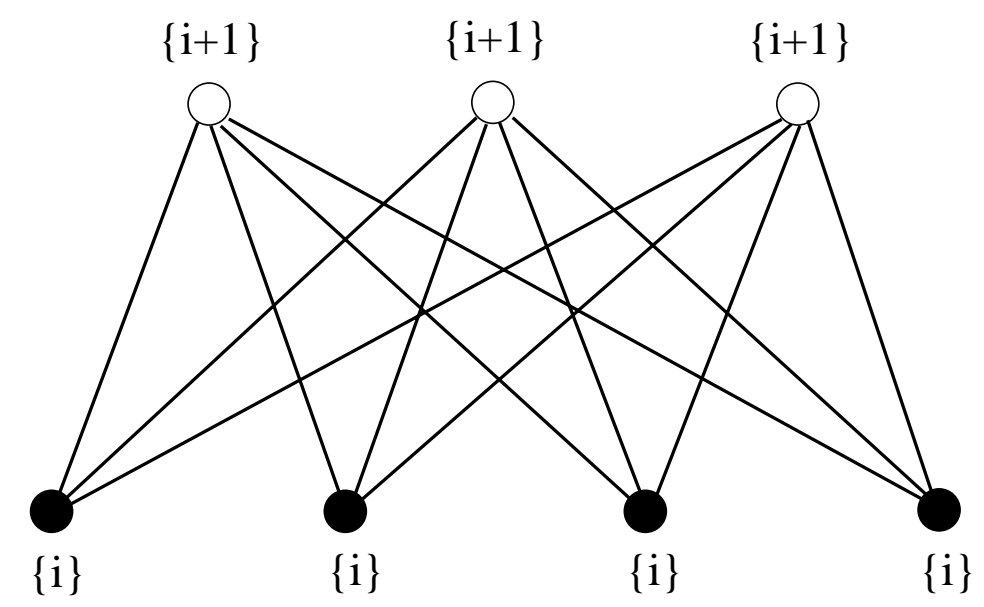

Figure 2.

Recall that the link of the vertex $x$ of $\Delta$ is the graph $L(x)$ such that:

- its vertices are the edges of $\Delta$ containing $x$;

- two vertices $J$ and $K$ of $L(x)$ are connected by an edge if a face of $\Delta$ contains both the edges represented by $J$ and $K$.

The link $L(x)$ is homeomorphic to the boundary of a little open contractible neighborhood of $x$, and the labeling of the edges of $\Delta$ containing $x$ induces a natural labeling of the vertices of $L(x)$.

If all the integers $q_{i}$ are equal, we say that $\Delta$ has constant thickness. 
We endow $\Delta$ with the length metric induced by its 2-cells. Properties (i), (ii), (iii) and (iv) above characterize $\Delta$ up to isometry (see [B1], proposition 2.2.1). The metric space $\Delta$ is geodesic and is a CAT $(-1)$ space (which means that its triangles are thinner than those of the hyperbolic plane with curvature -1 , see $[\mathrm{Ba}])$. This follows from a criterion of $\mathrm{W}$. Ballmann $[\mathrm{Ba}]$, from the contractibility of $\Delta$, and from the description of the links of $\Delta$ (properties (i) and (iv) above).

Denote by $\operatorname{Isom}(\Delta)$ the group of isometries of $\Delta$. This group is locally compact (for the topology of uniform convergence on compact subsets of $\Delta$ ). Let $\Gamma$ be the fundamental group of the orbihedron described above. It admits the following presentation

$$
\Gamma=\left\langle s_{i}, i \in \mathbf{Z} / r \mathbf{Z} \mid s_{i}^{q_{i}+1}=1,\left[s_{i}, s_{i+1}\right]=1\right\rangle .
$$

By construction, $\Gamma$ acts simply transitively on the set of 2-cells of $\Delta$. Hence, $\Gamma$ is a co-compact lattice of $\operatorname{Isom}(\Delta)$. Other examples of lattices of $\operatorname{Isom}(\Delta)$ are described in $[\mathrm{H}]$, [GP], [R], [B2].

Note that the subgroup of $\operatorname{Isom}(\Delta)$ preserving the labeling of $\Delta$ is a simple, non linear, uncountable and normal subgroup of finite index, by a theorem of $\mathrm{F}$. Haglund and F. Paulin [HP].

\subsection{Building structure and trees-walls}

In this section, we describe the basic notions of building theory in the special setting of buildings $\Delta$ introduced in 1.1. See [Br] and [Ro] for complete treatment about buildings. We also define the notion of «trees-walls》 which plays a crucial role in sections 2.1 and 2.2. This notion was introduced in [B1] 2.4.A, and is particular to buildings with right angles.

A. Chambers and apartments. 2-cells of $\Delta$ will be called chambers. Let $W$ be the co-compact Fuchsian Coxeter group generated by inversions in the edges of $R$ (see [Ma], IV.H.11). The group $W$ possesses the following presentation:

$$
W=\left\langle s_{i}, i \in \mathbf{Z} / r \mathbf{Z} \mid s_{i}^{2}=1,\left[s_{i}, s_{i+1}\right]=1\right\rangle,
$$

and generates a tiling (denoted by $W R$ ) of $H_{\mathbf{R}}^{2}$ by copies of $R$. Its edges and its vertices are labeled in the same manner as in 1.1. An apartment of $\Delta$ is a labeled cell sub-complex of $\Delta$, which is isomorphic to the labeled 2-complex $W R$. Apartments are totally geodesic in $\Delta$ (this can be seen in the links). An abstract theorem of J. Tits (see [Ro], theorem 4.9), together with properties 1.1 (i) to (iv) give the following result.

Proposition. The 2-complex $\Delta$ is a Tits building, in other words $\Delta$ satisfies the following properties: 
(i) two chambers of $\Delta$ are always contained in an apartment of $\Delta$;

(ii) if $A$ and $A^{\prime}$ are two apartments of $\Delta$ whose intersection is not empty, there exists an isomorphism of labelled cell complexes $\phi: A \rightarrow A^{\prime}$ fixing $A \cap A^{\prime}$.

B. Retractions. Let $A$ be an apartment of $\Delta$ and let $c$ be a chamber of $A$. The retraction from $\Delta$ onto $A$ centered at $c$ is the morphism of labeled cell complexes $\rho: \Delta \rightarrow A$ defined as follows. Let $d$ be a chamber of $\Delta$. By the previous proposition, part (i), there exists an apartment $B$ containing $c$ and $d$. Moreover, by the same proposition, part (ii), there exists an isomorphism of labeled cell complexes $\phi: B \rightarrow A$ fixing $B \cap A$. We let $\rho(d)=\phi(d)$.

C. Types, walls and trees-walls. From now on, we call type of a vertex or of an edge of $\Delta$ its label. A wall of $\Delta$ (respectively of an apartment $A$ of $\Delta$ ) is a bi-infinite geodesic contained in the 1-skeleton of $\Delta$ (respectively of $A$ ). The description of the links of the vertices of $\Delta$ shows that the edges of a wall $m$ have all the same type that we call the type of $m$.

Consider now a complete bipartite graph $L$ whose vertices are labeled by $\{s\}$ and $\{t\}$. We say that two vertices of $L$ are diametrically opposite if their simplicial distance is equal to 2 . The relation on the set of vertices of $L$ defined by «two vertices are equivalent if they are diametrically opposite or equal» is an equivalence relation and has two equivalence classes, namely the set of vertices labeled by $\{s\}$ and the set of vertices labeled by $\{t\}$.

Therefore, we obtain an equivalence relation on the set of edges of $\Delta$ as follows: two edges of $\Delta$ are equivalent if they are contained in a wall of $\Delta$. An equivalence class is called a tree-wall of $\Delta$. The edges of a tree-wall $T$ have all the same type that we call the type of $T$. Let $T$ be a tree-wall of type $\{i\}$. Then, we have the following properties:

(i) $T$ is a totally geodesic, bi-homogeneous tree in $\Delta$;

(ii) $T$ divides $\Delta$ into $q_{i}+1$ connected components;

(iii) Two distinct tree-walls share at most one vertex of $\Delta$ (since they are totally geodesic);

(iv) A geodesic line which intersects transversely a tree-wall intersects it at exactly one point (for the same reason as in (iii)).

\section{The boundary of $\Delta$}

Since $\Delta$ is a CAT $(-1)$ space, it possesses a boundary at infinity denoted by $\partial \Delta$ and defined as follows (see $[\mathrm{CDP}]$ or $[\mathrm{GH}]$ for more details). Recall that a geodesic ray of $\Delta$ is an isometric embedding $r:[0, \infty) \rightarrow \Delta$. The boundary of $\Delta$ is the set of geodesic rays $r$ so that $r(0)=x$ where $x$ is a base-point in $\Delta$. We endow $\partial \Delta$ with the topology of uniform convergence on compact subsets of $[0, \infty)$. By an argument of $\mathrm{N}$. Benakli $[\mathrm{Be}], \partial \Delta$ is homeomorphic to the Menger sponge (see 
$[\mathrm{A}])$.

In this part, we define some useful tools for the proof of the main theorem. Section 2.1 is devoted to the construction of combinatorial metrics on $\partial \Delta$, to the study of the induced conformal structure and to a characterization «à la Sullivan» of isometries of $\Delta$. In section 2.2, we investigate the structure of the set of boundaries of apartments in order to give nice properties of geodesics and curves on the boundary of $\Delta$. The Loewner structure of $\partial \Delta$ is discussed in section 2.3.

\subsection{Metric structures on $\partial \Delta$}

In this section, we define combinatorial metrics on $\partial \Delta$, and the associated notions of horospherical distance and of Gromov product (see [Led] or [Ham] for their Riemannian analogues). These notions have been introduced in [B1] 2.4, for buildings $\Delta$ with constant thickness. In subsections 2.1.A, 2.1.B, 2.1.C, 2.1.D we give their definitions and their basic properties. The conformal structure of $\partial \Delta$ is discussed in 2.1.E. In section 2.1.F we give a characterization of isometries of $\Delta$ based on Sullivan's ideas $[\mathrm{S}]$, which will be useful for the proof of the main theorem.

We first define a metric on the dual graph of $\Delta$ which depends on the thickness at each edge of $\Delta$.

A. Dual graph of $\Delta$. Let $\mathcal{G}(\Delta)$ be the dual graph of $\Delta$ : its vertices are centers of chambers of $\Delta$, they are connected by an edge if the corresponding chambers share an edge of $\Delta$. To each edge of $\mathcal{G}(\Delta)$ corresponds an edge of $\Delta$. If the type of this edge is $\{i\}$, we decide that the length of the corresponding edge in $\mathcal{G}(\Delta)$ is $\log q_{i}$. In such a way, we obtain a length metric on $\mathcal{G}(\Delta)$ that will be denoted by |. - .|. By property 1.1 (iv), it is left invariant under $\operatorname{Isom}(\Delta)$.

We are going to give an other useful formulation of this metric. Let $\mathcal{T}$ be the set of trees-walls of $\Delta$. For any tree-wall $T$ of type $\{i\}$, and any pair of chambers of $\Delta$ or points of $\partial \Delta$, denoted by $c_{1}, c_{2}$, we set $\alpha_{T}\left(c_{1}, c_{2}\right)=\log q_{i}$ if $c_{1}$ and $c_{2}$ belong to two distinct connected components of $(\Delta \cup \partial \Delta) \backslash(T \cup \partial T)$, and $\alpha_{T}\left(c_{1}, c_{2}\right)=0$ otherwise.

2.1.1. Proposition. For any pair $c$, $d$ of chambers of $\Delta$, we have

$$
|c-d|=\sum_{T \in \mathcal{T}} \alpha_{T}(c, d) .
$$

Proof. Consider a geodesic segment of $\Delta$ joining the interior of $c$ to the interior of $d$. We can assume that it does not contain any vertex of $\Delta$ (even if it means moving its endpoints). The tree-walls that it intersects are exactly those that separate $c$ and $d$. The chambers that it intersects define a continuous path in $\mathcal{G}(\Delta)$ whose endpoints are $c$ and $d$ and whose length is the right-hand side member of 2.1.1. Since any continuous path of $\mathcal{G}(\Delta)$ joining $c$ and $d$ must intersect the trees-walls that separate $c$ and $d$, the proof of proposition 2.1.1 is now complete. 
B. Horospherical distance. For three chambers $c, d$, e of $\Delta$, consider the function defined by

$$
N_{e}(c, d)=|c-e|-|d-e| .
$$

By proposition 2.1.1, we also have

$$
N_{e}(c, d)=\sum_{T \in \mathcal{T}} \alpha_{T}(c, e)-\alpha_{T}(d, e) .
$$

Let $\mathcal{T}(c, d)$ be the finite set of trees-walls separating the chambers $c$ and $d$. By a connectedness argument, the term $\alpha_{T}(c, e)-\alpha_{T}(d, e)$ in 2.1.2 is zero when $T$ belongs to $\mathcal{T} \backslash \mathcal{T}(c, d)$. Hence, for $\xi \in \partial \Delta$, the following quantity is well-defined (see [B1] for more details)

$$
N_{\xi}(c, d)=\sum_{T \in \mathcal{T}} \alpha_{T}(c, \xi)-\alpha_{T}(d, \xi) .
$$

We call it the combinatorial horospherical distance from $c$ to $d$ relatively to $\xi$. It satisfies the cocycle equality

$$
N_{\xi}(c, e)=N_{\xi}(c, d)+N_{\xi}(d, e) .
$$

Note that the combinatorial horospherical functions are locally constant on the complement in $\partial \Delta$ of endpoints of walls of $\Delta$. In the sequel, we will denote this set by $\partial_{\text {reg }} \Delta$; its elements will be called regular points of $\partial \Delta$.

C. Gromov product. If $c, d$ and $e$ are chambers in $\Delta$, their Gromov product is defined as

$$
\{d \mid e\}_{c}=\frac{1}{2}\{|c-d|+|c-e|-|d-e|\}=\frac{1}{2} \sum_{T \in \mathcal{T}} \alpha_{T}(c, d)+\alpha_{T}(c, e)-\alpha_{T}(d, e) .
$$

Denote by $\partial^{2} \Delta$ (respectively by $\left.\partial_{\text {reg }}^{2} \Delta\right)$ the set $(\partial \Delta)^{2}$ (respectively $\left.\left(\partial_{\text {reg }} \Delta\right)^{2}\right)$ minus the diagonal. For any chamber $c$ and any pair $d, e$ of chambers or points of $\partial \Delta$, let $\mathcal{T}(c, d, e)$ be the set of trees-walls $T$ so that the sets $\{c\}$ and $\{d, e\}$ are contained in two different connected components of $(\Delta \cup \partial \Delta) \backslash(T \cup \partial T)$. Note that the set $\mathcal{T}(c, d, e)$ is finite. By a connectedness argument, the general term in the sum above is zero for $T$ in $\mathcal{T} \backslash \mathcal{T}(c, d, e)$. Hence for $(\xi, \eta)$ in $\partial^{2} \Delta$, the following quantity is well-defined

$$
\{\xi \mid \eta\}_{c}=\frac{1}{2} \sum_{T \in \mathcal{T}} \alpha_{T}(c, \xi)+\alpha_{T}(c, \eta)-\alpha_{T}(\xi, \eta) .
$$

We call it the combinatorial Gromov product with base-point c. Note that $\{. \mid .\}_{c}$ is locally constant on $\partial_{\text {reg }}^{2} \Delta$. If we change the base-point, we have the following transformation rules (by (2.1.3) and (2.1.4))

$$
\{\xi \mid \eta\}_{c}=\{\xi \mid \eta\}_{d}+\frac{1}{2}\left(N_{\xi}(c, d)+N_{\eta}(c, d)\right)
$$


D. Combinatorial metrics on $\partial \Delta$. Let $A$ be an apartment of $\Delta$. Using a retraction, one sees that its dual graph is totally geodesic in $(\mathcal{G}(\Delta),|.-|$.$) . Choose$ a chamber $c$ as an origin and denote by $\mathcal{G}(A)$ the set of chambers of $A$. Set

$$
\tau=\limsup _{n \rightarrow 0}\left(\frac{1}{n} \log \#\{d \in \mathcal{G}(A) ;|c-d| \leq n\}\right) .
$$

Note that $\tau$ depends neither on $A$ nor on $c$.

For any chamber $c$ of $\Delta$, we now define a combinatorial metric $\delta_{c}$ on $\partial \Delta$. For this, we start with some definitions. Let $\xi \in \partial \Delta$ and let $r \in(0,+\infty)$. We define $B_{c}(\xi, r)$ as the set

$$
B_{c}(\xi, r)=\left\{\eta \in \partial \Delta: e^{-\tau\{\xi \mid \eta\}_{c}} \leq r\right\} .
$$

For a continuous path $\gamma$ in $\partial \Delta$, we define its «length» $l_{c}(\gamma)$ by

$$
\lim _{r \rightarrow 0} \inf \left\{\sum_{i} r_{i}\right\},
$$

where the infimum is taken over all the finite coverings $\left\{B_{c}\left(\xi_{i}, r_{i}\right)\right\}$ of $\gamma$ with $\xi_{i} \in \gamma$ and $r_{i} \leq r$. Finally, we set for $\xi, \eta \in \partial \Delta$,

$$
\delta_{c}(\xi, \eta)=\inf \left\{l_{c}(\gamma) ; \gamma \text { is a continuous path in } \partial \Delta \text { joining } \xi \text { and } \eta\right\} .
$$

The following result is proved in [B1] 3.1.D and in [B2] 2.2.7.

2.1.6. Proposition. The function $\delta_{c}$ is a length metric on $\partial \Delta$ and satisfies the following properties:

(i) There exists a constant $C \geq 1$ so that

$$
C^{-1} e^{-\tau\{\xi \mid \eta\}_{c}} \leq \delta_{c}(\xi, \eta) \leq C e^{-\tau\{\xi \mid \eta\}_{c}}
$$

whenever $\xi, \eta \in \partial \Delta$.

(ii) Let $H$ (respectively $\mathcal{H}_{c}$ ) be the Hausdorff dimension (respectively the Hausdorff measure) of $\left(\partial \Delta, \delta_{c}\right)$. Then we have $H=1+1 / \tau$. Moreover, $\left(\partial \Delta, \delta_{c}, \mathcal{H}_{c}\right)$ is an Ahlfors-regular space, this means that there exists a constant $C \geq 1$ so that

$$
C^{-1} r^{H} \leq \mathcal{H}_{c}(B) \leq C r^{H}
$$

whenever $B$ is a ball in $\partial \Delta$ whose radius $r$ is less that $\operatorname{diam}\left(\partial \Delta, \delta_{c}\right)$.

Remark. The number $\tau$ is the unique positive solution of the following equation (see [B2], 0.2):

$$
\sum_{i \in \mathbf{Z} / r \mathbf{Z}} \frac{q_{i}^{x}+q_{i+1}^{x}}{\left(1+q_{i}^{x}\right)\left(1+q_{i+1}^{x}\right)}=2 .
$$


In the special case where all the $q_{i}$ 's are equal to $q$, then

$$
\tau=\frac{\operatorname{Argch}((r-2) / 2)}{\log q} .
$$

E. Conformal structure of $\partial \Delta$. In this section, we will show that combinatorial metrics induce a conformal structure on $\partial \Delta$ which is invariant by $\operatorname{Isom}(\Delta)$. Recall that conformal homeomorphisms have been defined in 0.2 . Two metrics on the same space are said conformal if the identity map is a conformal homeomorphism (relatively to these metrics).

2.1.7. Proposition. (i) The combinatorial metrics on $\partial \Delta$ are pairwise conformal. More precisely, for $\xi \in \partial_{\text {reg }} \Delta$, we have

$$
\lim _{\eta \rightarrow \xi} \frac{\delta_{d}(\xi, \eta)}{\delta_{c}(\xi, \eta)}=e^{\tau N_{\xi}(c, d)}
$$

(ii) Any $g \in \operatorname{Isom}(\Delta)$ is a conformal homeomorphism of $\left(\partial \Delta, \delta_{c}\right)$. More precisely, whenever $\xi \in \partial_{\mathrm{reg}} \Delta$, we have

$$
\lim _{\eta \rightarrow \xi} \frac{\delta_{c}(g(\xi), g(\eta))}{\delta_{c}(\xi, \eta)}=e^{\tau N_{\xi}\left(c, g^{-1} c\right)}
$$

Proof. Note first that the complement of $\partial_{\text {reg }} \Delta$ in $\partial \Delta$ has zero measure. Indeed, the set of trees-walls is countable and the Hausdorff dimension of the boundary of a tree-wall is $1 / \tau$ which is a real number strictly less than the Hausdorff dimension of $\partial \Delta$. Since $\delta_{c}$ and $\delta_{d}$ are bilipschitz equivalent (see 2.1.6 (i) and (2.1.5)), they are quasi-symmetric equivalent. To show the equality in (i), apply (2.1.5) and note that the horospherical distance between $c$ and $d$ is constant in a neighborhood of a regular point of $\partial \Delta$. We complete the proof of (ii) by using (i) and the fact that $\delta_{c}(g(\xi), g(\eta))=\delta_{d}(\xi, \eta)$ whenever $d=g^{-1} c$.

F. A Sullivan's criterion. By the proof of proposition 2.1.7, for any chambers $c$ and $d$ of $\Delta$, we have

$$
\mathcal{H}_{d}(\xi)=e^{H \tau N_{\xi}(c, d)} \mathcal{H}_{c}(\xi)
$$

and for $g \in \operatorname{Isom}(\Delta)$,

$$
\left(g^{*} \mathcal{H}_{c}\right)(\xi)=e^{H \tau N_{\xi}\left(c, g^{-1} c\right)} \mathcal{H}_{c}(\xi) .
$$

$\left(g^{*} \mathcal{H}_{c}\right.$ is the pull-back measure of $\mathcal{H}_{c}$ by $\left.g\right)$.

It follows, from this and from (2.1.5), that the measure on $\partial^{2} \Delta$ defined by

$$
\mu(\xi, \eta)=e^{2 H \tau\{\xi \mid \eta\}_{c}} \mathcal{H}_{c}(\xi) \mathcal{H}_{c}(\eta)
$$


does not depend on the choice of $c$ and is invariant under the diagonal action of $\operatorname{Isom}(\Delta)$ on $\partial^{2} \Delta$.

2.1.9. Proposition. (i) Let $\Delta$ and $\Delta^{\prime}$ be two right-angled Fuchsian buildings and let $\mu$ and $\mu^{\prime}$ be the measures on $\partial^{2} \Delta$ and $\partial^{2} \Delta^{\prime}$ as defined in (2.1.8). Then, a homeomorphism $f: \partial \Delta \rightarrow \partial \Delta^{\prime}$ satisfies $(f \times f)_{*} \mu=C \mu^{\prime}$ (for some constant $C$ ) if and only if $f$ is the extension to the boundaries of an isometry $F: \Delta \rightarrow \Delta^{\prime}$.

(ii) Moreover, $\mu$ is $\Gamma$-ergodic for any co-compact lattice $\Gamma$ of $\operatorname{Isom}(\Delta)$.

This characterization of isometries is due to D. Sullivan in the case of noncompact rank 1 symmetric spaces (see [S], theorem 5). When $\Delta$ has constant thickness, it has been proved in [B1], proposition 4.4, as a consequence of a rigidity result for the combinatorial cross-ratio ([B1], proposition 2.4.7). This proof extends without any change to the setting of general right-angled Fuchsian buildings.

\subsection{Curves and geodesics on $\partial \Delta$}

In this section, we describe some geodesics of $\partial \Delta$ using boundaries of apartments (see proposition 2.2.1 below). Then, we give two results on the repartition of boundaries of apartments of $\Delta$. The first (proposition 2.2.2) is a topological one, the second (proposition 2.2.4) is a metric one. The results of this section will be used in the sequel as technical lemmas.

A. Geodesics in $\partial \Delta$. We fix a chamber $c$ in $\Delta$ and denote by $\delta$ and $\mathcal{H}$ the metric $\delta_{c}$ and the measure $\mathcal{H}_{c}$ respectively. Let $\mathcal{A}$ be the following set of boundaries of apartments:

$$
\mathcal{A}=\{a=\partial A: A \text { is an apartment containing } c\} .
$$

Since the retractions centered at $c$ increase the Gromov product $\{. \mid .\}_{c}$, they contract the metric $\delta$. Henceforth, elements of $\mathcal{A}$ are isometrically embedded in $(\partial \Delta, \delta)$.

2.2.1 Proposition. Any two points of $\partial \Delta$ are joined by a geodesic segment of $(\partial \Delta, \delta)$ which is the union of at most 4 geodesics segments contained in elements of $\mathcal{A}$.

Proof. Let $\xi$ and $\xi^{\prime}$ be two points of $\partial \Delta$ and let $r$ and $r^{\prime}$ be two geodesic rays of $\partial \Delta$ joining the interior of $c$ to $\xi$ and $\xi^{\prime}$ respectively. Even if it means moving the endpoint of $r^{\prime}$ in $c$, we can assume that $r^{\prime}$ does not pass through any vertex of $\Delta$. Let $A_{0}$ be an apartment of $\Delta$ containing $r$. If this apartment contains also $r^{\prime}$, the proof is complete. Suppose now that $r^{\prime}$ is not contained in $A_{0}$. Starting with $A_{0}$, by «bending》, we can construct an apartment $A_{\infty}$ containing $r^{\prime}$ as follows:

Let $t_{0}=\max \left\{t \in[0,+\infty] ; r^{\prime}(s) \in A_{0}\right.$ for $\left.s \in[0, t]\right\}$ and let $m_{0}$ be the wall of 
$A_{0}$ containing $r^{\prime}\left(t_{0}\right)$. By bending $A_{0}$ along $m_{0}$, we obtain an apartment $A_{1}$ which contains $r^{\prime}(t)$ for $t \in\left[0, t_{1}\right]$ with $t_{1}>t_{0}$. By induction, we construct sequences $\left(A_{k}\right),\left(m_{k}\right)$ and $\left(t_{k}\right)$ so that

(i) $A_{k}$ is an apartment of $\Delta$;

(ii) $m_{k}$ is a wall which is transversal to $r^{\prime}$ and which is contained in $A_{k}$ and $A_{k+1}$;

(iii) $\left(t_{k}\right)$ is increasing and satisfies

$$
\left[0, t_{k}\right]=\left\{t \in[0,+\infty] ; r^{\prime}(t) \in A_{k}\right\}, \quad r^{\prime}\left(t_{k}\right) \in m_{k} .
$$

The limit of the sequence of apartments $\left(A_{k}\right)$ is an apartment denoted by $A_{\infty}$ which contains $r^{\prime}$.

If no wall $m_{k}$ intersects $r$, then it is clear that $A_{\infty}$ contains $r$ and $r^{\prime}$ and the proof is complete. Otherwise, let $m_{K}$ be the first wall of the sequence $\left(m_{k}\right)$ which meets $r$. Then, the apartment $A_{K}$ contains $r$. Let $T$ be the tree-wall of $\Delta$ that contains $m_{K}$. Its boundary separates $\xi$ and $\xi^{\prime}$ in $\partial \Delta$. Indeed, a geodesic ray which intersects transversely a tree-wall shares with it exactly one point (see 1.2.C (ii), (iv)). Hence, any curve of $\partial \Delta$ joining $\xi$ and $\xi^{\prime}$ must meet $\partial T$. Therefore, our proposition follows from the following lemma.

Lemma. Let $\xi$ and $\xi^{\prime}$ be two points in $\partial \Delta$ and let $r$ be a geodesic ray connecting the interior of $c$ to $\xi$. Assume that $\xi^{\prime}$ is the endpoint of a wall $w$ of $\Delta$ that meets $r$. Then, there exists a geodesic segment of $\partial \Delta$ joining $\xi$ and $\xi^{\prime}$ which is the union of at most two geodesic segments contained in elements of $\mathcal{A}$.

Proof. Let $r^{\prime}$ be the half-wall of $w$ whose endpoints are $\xi^{\prime}$ and the point of intersection of $w$ and $r$. Let $A_{0}$ be an apartment which contains $r$ and let $A_{\infty}$ be an apartment which contains $r^{\prime}$ and which is obtained by the method described in the beginning of the proof of proposition 2.2.1. If there exists no wall $m_{k}$ (with the same notation as above) which intersects $r$, then $A_{\infty}$ contains $r$ and $r^{\prime}$, and the lemma is proved. Otherwise, let $K=\inf \left\{k \in \mathbf{N} ; m_{k}\right.$ meets $\left.r\right\}$. Denote by $m$ and $A$ the wall $m_{K}$ and the apartment $A_{K}$. Then, $A$ contains $r$ and $m$. Besides, the walls $m_{k}$ are pairwise disjoint because they are orthogonal to the half-wall $r^{\prime}$. Hence, $A_{\infty}$ contains all of them. In particular, it contains $m$. Let $D_{c}$ (respectively $D_{\xi^{\prime}}$ ) be the half-apartment of $A$ (respectively $A_{\infty}$ ) delimited by $m$ and which contains $c$ (respectively $\xi^{\prime}$ ). Then, $A^{\prime}=D_{c} \cup D_{\xi^{\prime}}$ is an apartment which contains $c$ and $\xi^{\prime}$.

To finish the proof of the lemma, we should show that there exists a geodesic segment of $\Delta$ joining $\xi$ and $\xi^{\prime}$ and which is contained in $\partial A \cup \partial A^{\prime}$. To do this, consider a geodesic segment $S$ of $\partial \Delta$ joining $\xi$ and $\xi^{\prime}$. The tree-wall $T$ which contains $m$ separates $\xi$ from $\xi^{\prime}$ in $\partial \Delta$. Hence, $S$ meets $\partial T$ in at least one point $\eta$. Let $\gamma$ (respectively $\gamma^{\prime}$ ) be the part of $S$ between $\xi$ and $\eta$ (respectively $\xi^{\prime}$ and $\eta$ ) and let $\rho$ (respectively $\rho^{\prime}$ ) be the retraction centered at $c$ from $\Delta$ onto $A$ (respectively $\left.A^{\prime}\right)$. Since retractions preserve types, we have

$$
\rho(T \cup \partial T)=\rho^{\prime}(T \cup \partial T)=m \cup \partial m .
$$


Moreover, restricted to $T \cup \partial T, \rho$ is equal to $\rho^{\prime}$. It follows that $\rho(\gamma) \cup \rho^{\prime}\left(\gamma^{\prime}\right)$ is a continuous path in $\partial A \cup \partial A^{\prime}$ joining $\xi$ and $\xi^{\prime}$. Its length is less than those of $S$, since $\rho$ and $\rho^{\prime}$ contract the metric $\delta$. Hence, $\rho(\gamma) \cup \rho^{\prime}\left(\gamma^{\prime}\right)$ is a geodesic curve and the proof is complete.

B. Approximation of curves in $\partial \Delta$. For $\xi \in \partial \Delta$, set

$$
\mathcal{A}_{\xi}=\{a \in \mathcal{A}: \xi \in a\}
$$

The proposition below «approximates» the curves in $\partial \Delta$ containing $\xi$, by elements of $\mathcal{A}_{\xi}$.

2.2.2 Proposition. For $\mathcal{H}$-almost every $\xi$ in $\partial \Delta$, the set $\mathcal{A}_{\xi}$ has the following property:

$(\mathrm{P})$ if $\gamma:[0,1] \rightarrow \partial \Delta$ is a continuous curve with $\gamma(0)=\xi$, then, for any $t \in] 0,1]$, the sub-curve $\gamma(] 0, t])$ intersects at least one element of $\mathcal{A}_{\xi}$.

Let $N$ be the complement in $\partial \Delta$ of the set of points $\xi$ satisfying property (P). We should show that the measure of $N$ is 0 . We begin with a geometric characterization of elements of $N$.

Lemma. Let $\xi \in \partial A$ where $A$ is an apartment containing $c$. Then, $\xi$ belongs to $N$ if and only if there exists a sequence $\left(s_{k}\right)_{k \in \mathbf{N}}$ of vertices of $A$ which tends to $\xi$ so that

(i) each geodesic segment $\left[s_{k}, s_{k+1}\right], k \in \mathbf{N}$, belongs to a wall $m_{k}$ of $A$;

(ii) the walls $m_{k}$ separate $c$ from $\xi$;

(iii) For every $k \in \mathbf{N}^{*}, s_{k-1} \widehat{s_{k}} s_{k+1}= \pm \pi / 2$ and $s_{k-1} \widehat{s_{k}} s_{k+1}=-s_{k} \widehat{s_{k+1}} \widehat{s}_{k+2}$.

Proof. Suppose that $\xi$ belongs to $N$. Let $\gamma:[0,1] \rightarrow \partial \Delta$ be a curve with $\gamma(0)=\xi$ and so that $a \cap \gamma(10,1])=\emptyset$ whenever $a \in \mathcal{A}_{\xi}$.

Let $r$ be a geodesic ray of $A$ joining the interior of $c$ to $\xi$. As usual, we can assume that $r$ does not meet any vertex of $A$. Since $\gamma(1)$ is different from $\xi$, there exists a tree-wall $T_{0}$ which is transversal to $r$ and whose boundary separates $\xi$ and $\gamma(1)$. Hence, $\gamma$ meets $\partial T_{0}$ at time $t_{0}<1$. Let $x_{0}$ be the point of intersection of $T_{0}$ and $r$, and let $r_{0}$ be the half-wall $\left[x_{0}, \gamma\left(t_{0}\right)\right)$ of $T_{0}$. Since $\gamma\left(t_{0}\right)$ does not belong to any element of $\mathcal{A}_{\xi}$, there exists a tree-wall $T_{1}$ which is transversal to $r$ and $r_{0}$ and whose boundary separates $\xi$ and $\gamma\left(t_{0}\right)$ (see the proof of proposition 2.2.1). Hence, $\gamma$ meets $\partial T_{1}$ at time $t_{1}<t_{0}$. We repeat this process, and we obtain sequences $\left(t_{n}\right),\left(T_{n}\right)$, where $\left(t_{n}\right)$ is a decreasing sequence in $[0,1]$ and $T_{n}$, for any $n \in \mathbf{N}$, is a tree-wall which meets $r$ so that $\gamma\left(t_{n}\right) \in \partial T_{n}, T_{n} \neq T_{n+1}$ and $T_{n} \cap T_{n+1} \neq \emptyset$. Using the continuity of $\gamma$, we see that $t_{k}$ tends to 0 and therefore, the sequence of trees-walls $T_{n}$ tends to $\xi$.

Consider now the images of $T_{n}, n \in \mathbf{N}$, by the retraction centered at $c$ from $\Delta$ onto $A$. The set of these images is a collection of walls of $A$ which intersect 


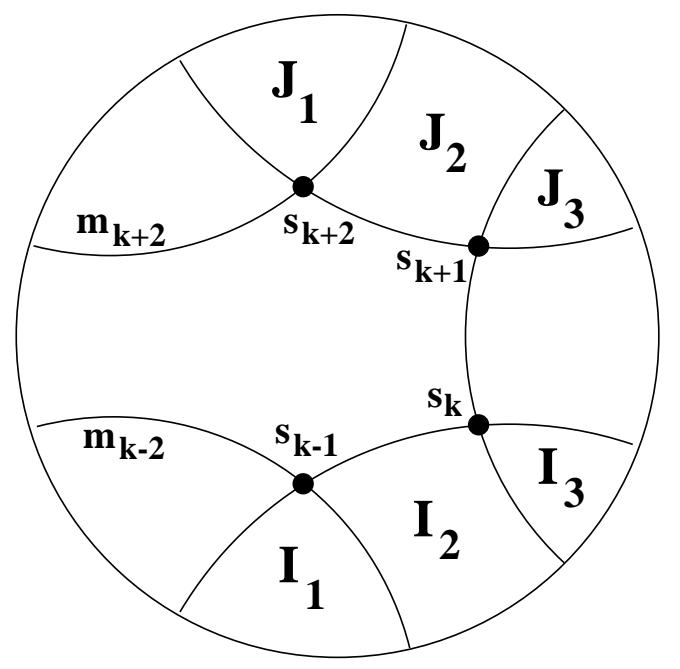

Figure 3.

$r$. Their union, denoted by $\mathcal{R}$, contains a continuous path which tends to $\xi$. It follows that $\mathcal{R}$ contains a sequence of vertices $\left(s_{k}\right)$ which tends to $\xi$ and which satisfies the assertions (i) and (ii) of the lemma.

We have now to prove (iii). By passing to a subsequence of $\left(s_{k}\right)$, we can assume that $m_{k}$ is different from $m_{k+1}$ and $m_{k+2}$. Then, for $k \in \mathbf{N}^{*}, s_{k-1} \widehat{s_{k} s_{k+1}}=$ $\pm \pi / 2$. Even if this means to change $s_{0}$ by its symmetric relatively to $s_{1}$, we can also assume that $\widehat{s} 0_{0} \widehat{s}_{1}=-s_{1} \widehat{s_{2} s_{3}}$ and we would like to show $(*) s_{k-1} \widehat{s_{k} s_{k+1}}=$ $-s_{k} \widehat{s} k+1_{k+2}$ whenever $k \geq 2$. Suppose that the relation $(*)$ is not satisfied for some $k$ and consider the domains $I_{n}, J_{n}, n=1,2,3$ defined as in figure 3 . Since $r$ meets the walls $m_{k-1}$ and $m_{k+1}, r$ has an endpoint $a$ (respectively $b$ ) in the half-apartment $I_{1} \cup I_{2} \cup I_{3}$ (respectively $J_{1} \cup J_{2} \cup J_{3}$ ). Since $r$ meets $m_{k-2}, m_{k-1}$ and $m_{k}, a$ belongs to $I_{1}$ and $b$ belongs to $J_{3}$. Since $r$ meets $m_{k}, m_{k+1}$ and $m_{k+2}$, $a$ belongs to $I_{3}$ and $b$ belongs to $J_{1}$. This is a nonsense, and implication $(\Rightarrow)$ of the lemma is proved.

The proof of the converse (that we won't use in the sequel) is left to the reader as an exercise.

We now prove proposition 2.2.2.

Proof of proposition 2.2.2. A continuous path $\gamma$ is called alternate if $\gamma$ is a simple path which is parameterized by arc length, which is contained in the 1-skeleton of an apartment of $\Delta$ and which turns alternately on the left and on the right. Given a vertex $s$ of $\Delta$, denote by $\Lambda_{s}$ the set of endpoints in $\partial \Delta$ of alternate paths beginning at $s$. It is a closed subset in $\partial \Delta$ with empty interior. To see this, consider an apartment $A$ of $\Delta$ containing $s$. The union of alternate paths starting at $s$ and contained in $A$ is a quasi-convex tree in $A$ (see figure 4 ). The set of their 


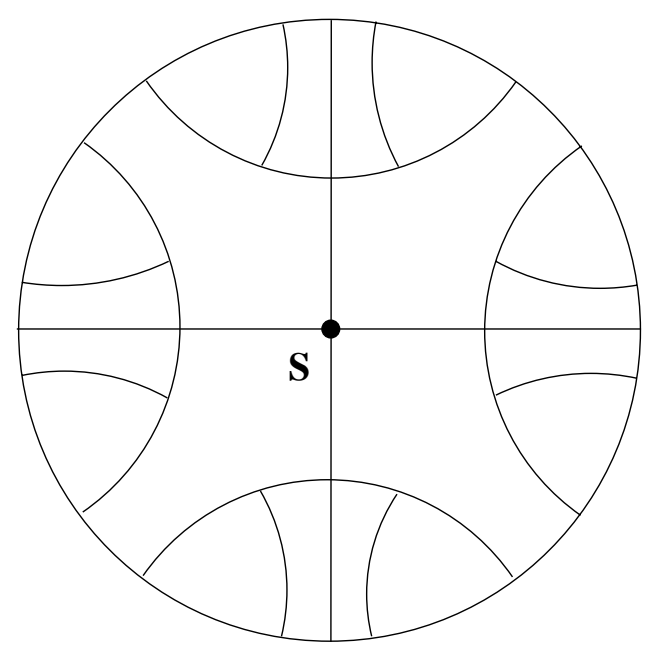

Figure 4.

endpoints is a Cantor set contained in $\partial A$ that will be denoted by $\Lambda_{s, A}$. Using proposition 1.2.A (ii), we see that

$$
\Lambda_{s} \cap \partial A=\Lambda_{s, A}
$$

Therefore, $\Lambda_{s}$ has empty interior in $\partial \Delta$.

Set $\Lambda=\bigcup_{s} \Lambda_{s}$ where $s$ runs over all the vertices of $\Delta$. By the previous lemma, $N$ is contained in $\Lambda$. We are going to show that the measure of $\Lambda$ is 0 . Since $\Lambda$ is invariant under the action of $\operatorname{Isom}(\Delta)$ and since the measure $\mathcal{H} \times \mathcal{H}$ is ergodic under the diagonal action of $\operatorname{Isom}(\Delta)$ on $\partial^{2} \Delta$ (see proposition 2.1 .9 (ii)), it is enough to prove that $\partial^{2} \Delta$ doesn't contain any dense orbit of pair of distinct points of $\Lambda$.

Let $\Theta$ be the orbit of a pair $\left(\xi_{+}, \xi_{-}\right)$of distinct points of $\Lambda$. Let $\mathcal{C}_{+}$and $\mathcal{C}_{-}$be alternate paths which converge to $\xi_{+}$and $\xi_{-}$respectively. There exists $R \geq 0$ such that the distance from any point of the geodesic $\left(\xi_{-} \xi_{+}\right)$to the set of vertices of $\mathcal{C}_{-} \cup \mathcal{C}_{+}$is less than $R$. Denote by $\mathcal{S}_{R}$ the (finite) set of vertices of $\Delta$ whose distance to $c$ is less than $R$ and denote by $U$ the open subset of $\partial^{2} \Delta$ whose elements are the pairs $(\xi, \eta)$ so that the geodesic $(\xi \eta)$ passes through $c$. Then, we have

$$
\Theta \cap U \subset\left(\bigcup_{s \in \mathcal{S}_{R}} \Lambda_{s} \times \partial \Delta\right) \cup\left(\partial \Delta \times \bigcup_{s \in \mathcal{S}_{R}} \Lambda_{s}\right)
$$

However, the right-hand side member is a closed subset with empty interior. Hence, $\Theta$ is not a dense orbit in $\partial^{2} \Delta$.

C. Pencils of boundaries of apartments. Let $A_{0}$ be an apartment containing $c$ and denote by $a_{0}$ its boundary. We denote also by $\rho$ the contracting map 
from $\partial \Delta$ onto $a_{0}$ which is induced by the retraction $\rho$ centered at $c$ from $\Delta$ onto $A_{0}$. The compact subgroup $K$ of $\operatorname{Isom}(\Delta)$ which stabilizes point-wise the chamber $c$ preserves any fiber of $\rho$. The argument given in [B1] 2.3.B shows that it acts transitively on $\mathcal{A}$. Therefore, it acts transitively on any fiber of $\rho$. For $\zeta \in a_{0}$, denote by $\nu_{\zeta}$ the probability measure on $\rho^{-1}(\zeta)$ induced by the Haar measure of $K$. Let $d l$ be the 1-Hausdorff measure of the length space $\left(a_{0}, \delta\right)$. The following result is proved in [B2] 2.2.4 and 2.2.7.

2.2.3. Proposition. (i) The spaces $\left(\rho^{-1}(\zeta), \delta, \nu_{\zeta}\right), \zeta \in a_{0}$, are uniformly Ahlfors-regular of dimension $H-1$, this means that there exists $C \geq 1$ depending only on $\Delta$ such that

$$
C^{-1} r^{H-1} \leq \nu_{\zeta}\left(B \cap \rho^{-1}(\zeta)\right) \leq C r^{H-1}
$$

whenever $\zeta \in a_{0}, B$ is a ball of $(\Delta, \delta)$ centered in $\rho^{-1}(\zeta)$ and of radius $r \leq 1$. $\partial \Delta$,

(ii) There exists $C \geq 1$ depending only on $\Delta$ such that for any Borel set $B$ of

$$
C^{-1} \mathcal{H}(B) \leq \int_{a_{0}} \nu_{\zeta}\left(B \cap \rho^{-1}(\zeta)\right) d l(\zeta) \leq C \mathcal{H}(B) .
$$

For two points $\xi$ and $\eta$ in $a_{0}$, we call pencil joining $\xi$ and $\eta$ the set $\mathcal{A}_{\xi, \eta}=$ $\mathcal{A}_{\xi} \cap \mathcal{A}_{\eta}$. The following result which gives an estimate of the size of $\mathcal{A}_{\xi, \eta}$ in each fiber of $\rho$, generalizes and improves the key lemma (lemma 4 ) of [BP]. For $\zeta$ in $a_{0}$, consider the closed subset of $\rho^{-1}(\zeta)$ defined by

$$
F_{\xi, \eta}^{\zeta}=\rho^{-1}(\zeta) \cap \bigcup_{a \in \mathcal{A}_{\xi, \eta}} a .
$$

2.2.4. Proposition. (i) There exists a constant $C \geq 1$ depending only on $\Delta$ such that

$$
C^{-1}\left(\phi_{\xi, \eta}(\zeta)\right)^{H-1} \leq \nu_{\zeta}\left(F_{\xi, \eta}^{\zeta}\right) \leq C\left(\phi_{\xi, \eta}(\zeta)\right)^{H-1}
$$

where $\phi_{\xi, \eta}(\zeta)=\inf (\delta(\zeta, \xi), \delta(\zeta, \eta))$.

(ii) The spaces $\left(F_{\xi, \eta}^{\zeta}, \delta, \nu_{\zeta}\right)$ are uniformly Ahlfors-regular with dimension $H-1$ : there exists $C \geq 1$ depending only on $\Delta$ such that for every ball $B$ whose center belongs to $F_{\xi, \eta}^{\zeta}$ and whose radius $r$ is less than $\phi_{\xi, \eta}(\zeta)$, we have

$$
C^{-1} r^{H-1} \leq \nu_{\zeta}\left(B \cap F_{\xi, \eta}^{\zeta}\right) \leq C r^{H-1} .
$$

Proof. We first show (i). The right-hand side inequality follows from proposition 2.2.3(i), since by the triangle inequality, we have

$$
F_{\xi, \eta}^{\zeta} \subset B\left(\zeta, 2 \phi_{\xi, \eta}(\zeta)\right) \cap \rho^{-1}(\zeta)
$$


Before proving the other inequality, we start with some reductions. Let $\mathcal{C}_{\zeta}$ be the connected component of $a_{0} \backslash\{\xi, \eta\}$ containing $\zeta$. If $\xi^{\prime}$ and $\eta^{\prime}$ are two other points of $\mathcal{C}_{\zeta}$ separated by $\zeta$, then

$$
F_{\xi^{\prime}, \eta^{\prime}}^{\zeta} \subset F_{\xi, \eta}^{\zeta}
$$

To see this, consider $a^{\prime} \in \mathcal{A}_{\xi^{\prime}, \eta^{\prime}}$. By gluing together the connected component of $a^{\prime} \backslash\left\{\xi^{\prime}, \eta^{\prime}\right\}$ which meets $\rho^{-1}(\zeta)$ with the connected component of $a_{0} \backslash\left\{\xi^{\prime}, \eta^{\prime}\right\}$ which does not contain $\zeta$, we obtain an element $a$ of $\mathcal{A}_{\xi, \eta}$ so that $a^{\prime} \cap \rho^{-1}(\zeta)=a \cap \rho^{-1}(\zeta)$. It follows that it is enough to show the left-hand side inequality under the following assumptions:

(a) $\delta(\zeta, \xi)=\delta(\zeta, \eta) \leq \frac{\operatorname{diam}\left(a_{0}, \delta\right)}{4}$;

(b) the geodesic $(\xi \eta)$ of $A_{0}$ separates $\zeta$ from $c$.

To simplify, denote $F_{\xi, \eta}^{\zeta}$ by $F$. Let us describe the geometry of $F$ and $\rho^{-1}(\zeta)$. Let $x$ be a point in the interior of $c$ and denote by $r_{\xi}, r_{\eta}, r$ respectively the geodesic rays $[x \xi),[x \eta),[x \zeta)$ of $A_{0}$. Even if this means to move $x$ in $c$, we can assume that these rays do not contain any vertex of $\Delta$. The set $\rho^{-1}(r)$ is a tree whose root is $x$. The edges of $\Delta$ that it intersects induce a $\rho$-invariant labeling of its vertices. The valency of a vertex of type $\{i\}$ is $q_{i}+1$. The set $\rho^{-1}(\zeta)$ is the boundary of the tree $\rho^{-1}(r)$.

Let $\omega$ be a point in $\rho^{-1}(\zeta)$ and denote by $r_{\omega}$ the geodesic ray $[x \omega)$ of $\rho^{-1}(r)$. This ray can be constructed by the «bending 》 method described in the proof of proposition 2.2.1. In such a way, we see that $\omega$ belongs to $F$ if and only if no wall along which we bend intersects $r_{\eta}$ nor $r_{\eta}$.

Let $s_{1}, \ldots, s_{l}$ be the vertices of $r$ through which passes a wall of $A_{0}$ intersecting $r_{\xi}$ or $r_{\eta}$ and denote by $\left\{i_{1}\right\}, \ldots,\left\{i_{l}\right\}$ their types. Then, $F$ is the boundary of a sub-tree of $\rho^{-1}(r)$ whose root is $x$ and whose vertices $s$ have valency 2 if $\rho(s)$ belongs to $\left\{s_{1}, \ldots, s_{l}\right\}$ and $q_{i}+1$ (where $\{i\}$ is the type of $s$ ) otherwise. Hence, since the group $K$ acts on the tree $\rho^{-1}(r)$ and acts transitively on its boundary, we obtain

$$
\nu_{\zeta}(F)=\left(q_{i_{1}} \ldots q_{i_{l}}\right)^{-1} .
$$

Because the points $\xi, \eta, \zeta$ and the chamber $c$ are all in $A_{0} \cup \partial A_{0}$, the relation (2.1.4) and the assumption (b) show that $\nu_{\zeta}(F)$ is equal to $e^{-N}$, where

$$
N=\{\xi \mid \zeta\}_{c}+\{\eta \mid \zeta\}_{c}-\{\xi \mid \eta\}_{c} .
$$

It follows from proposition 2.1.6

$$
\nu_{\zeta}(F) \geq C^{-1}\left(\frac{\delta(\xi, \zeta) \delta(\eta, \zeta)}{\delta(\xi, \eta)}\right)^{H-1}
$$

where $C$ is a constant depending only on $\Delta$. Moreover, assumption (a) implies $\delta(\xi, \zeta)=\delta(\eta, \zeta)$ and $\delta(\xi, \eta)=2 \delta(\xi, \zeta)$. This completes the proof of (i). 
We now prove (ii). Let $r \in \mathbf{R}$ such that $0<r<\phi_{\xi, \eta}(\zeta)$. By a classical covering theorem (see for instance [Mat], theorem 2.1), there exists a finite collection of balls $\left\{B_{i}, i \in I\right\}$ of $(\partial \Delta, \delta)$, whose centers are on $F$ and whose radius is $r$, so that

$$
F \subset \bigcup_{i \in I} B_{i}
$$

and so that the balls $\frac{1}{5} B_{i}, i \in I$, are pairwise disjoint $\left(\frac{1}{5} B_{i}\right.$ is the ball whose center is the same as $B_{i}$ and whose radius is $\left.r / 5\right)$. Since $K$ acts transitively on $\mathcal{A}$ preserving fibers of $\rho$, the stabilizer of $\xi$ and $\eta$ in $K$ acts transitively on $\mathcal{A}_{\xi, \eta}$ and therefore acts transitively by isometries on $(F, \delta)$. Hence, by inclusion $(2)$, we obtain, for every ball $B$ of $(\partial \Delta, \delta)$ whose center is on $F$ and whose radius is $r$,

$$
\nu_{\zeta}(F \cap B) \geq \frac{\nu_{\zeta}(F)}{|I|} .
$$

Moreover, we have by (1)

$$
\nu_{\zeta}\left(\bigcup_{i \in I} \frac{1}{5} B_{i} \cap \rho^{-1}(\zeta)\right) \leq \nu_{\zeta}\left(B\left(\zeta, 3 \phi_{\xi, \eta}(\zeta)\right) \cap \rho^{-1}(\zeta)\right)
$$

and then, by proposition 2.2.3 (i),

$$
|I| \leq C \frac{\left(\phi_{\xi, \eta}(\zeta)\right)^{H-1}}{r^{H-1}}
$$

where $C$ is a constant depending only on $\Delta$. Using this last inequality, inequality (3) and proposition 2.2.4 (i), we obtain the left-hand side inequality of (ii). The reverse inequality follows easily from proposition 2.2 .3 (i).

\subsection{Absolute continuity, Loewner spaces}

According to $[\mathrm{BP}]$ theorem 1 , the metric space $(\partial \Delta, \delta)$ admits Poincaré inequalities when $\Delta$ has constant thickness. This result can be extended to all right-angled Fuchsian buildings by proposition 2.2.4 (i) which is a generalization of the main lemma (lemma 4) of [BP].

Existence of Poincaré inequalities on a metric space has important consequences as showed by J. Heinonen and P. Koskela in [HK]. Here, we explain some of these which will be useful in the proof of our main theorem: strong properties of absolute continuity of quasi-symmetric homeomorphisms and Loewner space structure of $\partial \Delta$.

A. Absolute continuity. Let $Y$ be an Ahlfors-regular metric space and let $f$ be a quasi-symmetric homeomorphism from $\partial \Delta$ to $Y$. We denote by Hdim the Hausdorff dimension. 
2.3.1. Proposition. (i) We have $\operatorname{Hdim}(\partial \Delta) \leq \operatorname{Hdim}(Y)$;

(ii) If $\operatorname{Hdim} \partial \Delta=\operatorname{Hdim}(Y)$, then $f$ and $f^{-1}$ are absolutely continuous with respect to the Hausdorff measures of $\partial \Delta$ and $Y$.

Proof. The first assertion follows from [B2] theorem 2.1, or more generally from $[\mathrm{T}]$ corollary 1.7, the second assertion follows from [HK] corollary 7.13.

With the same notations as in 0.2 , one gets the

2.3.2 Corollary. If $\operatorname{Hdim}(\partial \Delta)=\operatorname{Hdim}(Y)$, then for $\mathcal{H}$-almost all $\xi \in \partial \Delta, l_{f}(\xi)$ and $L_{f}(\xi)$ belong to $(0,+\infty)$.

Proof. Let $\phi$ be the homeomorphism of quasi-symmetry of $f$ (see 0.2 ). Then we have

$$
l_{f} \leq L_{f} \leq \phi(1) l_{f} .
$$

Assume that $\operatorname{Hdim}(\partial \Delta)=\operatorname{Hdim}(Y)=H$. Proposition 2.3.1 (ii) implies that the derivative $\mu_{f}$ of $f^{*} \mathcal{H}_{Y}$ with respect to $\mathcal{H}$ belongs almost surely to $(0,+\infty)$. Moreover, for almost every $\xi \in \partial \Delta$, we have by [Fe] 2.9,

$$
\mu_{f}(\xi)=\lim _{r \rightarrow 0} \frac{\mathcal{H}_{Y}(f(B(\xi, r)))}{\mathcal{H}(B(\xi, r))}
$$

Hence, there exists a constant $C \geq 1$ depending only on the regularity constants of $\mathcal{H}$ and $\mathcal{H}_{Y}$ such that

$$
C^{-1} l_{f}^{H} \leq \mu_{f} \leq C L_{f}^{H} \text { a.e. . }
$$

Inequalities (1) and (2) show that $l_{f}(\xi)$ and $L_{f}(\xi)$ belong almost surely to $(0, \infty)$.

The next result means that $f$ is absolutely continuous along almost every rectifiable curves. The definition of the modulus of a family of curves is recalled in the next subsection.

2.3.3. Proposition. If $\operatorname{Hdim}(\partial \Delta)=\operatorname{Hdim}(Y)$, the modulus of the family of all rectifiable curves $\gamma$ of $\partial \Delta$ along which $f \circ \gamma$ is not absolutely continuous is 0 .

Proof. See $[\mathrm{HK}]$ theorem 8.1 or $[\mathrm{T}]$ corollary 1.8.

B. Moduli. Loewner spaces. Let $X$ be a metric space. For simplicity, we assume that $X$ is Ahlfors regular with dimension $H>1$. Let $\mathcal{F}$ be a family of curves in $X$. The modulus of $\mathcal{F}$ is

$$
\operatorname{Mod}(\mathcal{F})=\inf \left\{\int_{X} u^{H} d \mathcal{H}_{X}\right\},
$$


where the infimum is taken over all $\mathcal{F}$-admissible functions $u$, namely measurable functions $u: X \rightarrow[0,+\infty]$ so that, for every rectifiable curve $\gamma$ in $\mathcal{F}, \int_{\gamma} u d s \geq 1$. If no curve in $\mathcal{F}$ is rectifiable, we set $\operatorname{Mod}(\mathcal{F})=0$.

If $E$ and $F$ are two continua in $X$, we denote by $\operatorname{Mod}(E, F)$ the modulus of the family of all curves of $X$ joining $E$ to $F$. The modulus admits the following nice properties (see [HK] sections 2 and 3 for more details):

(a) Mod is an exterior measure on the set of families of curves of $X$, namely

$$
\begin{gathered}
\operatorname{Mod}\left(\mathcal{F}_{1}\right) \leq \operatorname{Mod}\left(\mathcal{F}_{2}\right) \text { if } \mathcal{F}_{1} \subset \mathcal{F}_{2} \\
\operatorname{Mod}\left(\cup_{i=1}^{\infty} \mathcal{F}_{i}\right) \leq \sum_{i=1}^{\infty} \operatorname{Mod}\left(\mathcal{F}_{i}\right)
\end{gathered}
$$

(b) for $0 \leq r \leq 2 R$, we have

$$
\operatorname{Mod}(\bar{B}(x, r), X \backslash B(x, R)) \leq(\log R / r)^{1-H},
$$

where $C$ is a constant depending only on $X$.

For two non-degenerate and disjoint continua $E$ and $F$ of $X$, we set

$$
\Omega(E, F)=\frac{\inf \{\operatorname{diam} E, \operatorname{diam} F\}}{\operatorname{distance}(E, F)} .
$$

Definition. The metric space $X$ is a Loewner space if there exists a non decreasing homeomorphism $\psi:(0,+\infty) \rightarrow(0,+\infty)$ so that for every non degenerate and disjoint continua $E$ and $F$ of $X$, we have

$$
\operatorname{Mod}(E, F) \geq \psi(\Omega(E, F)) .
$$

2.3.4. Proposition. The metric space $(\partial \Delta, \delta)$ is a Loewner space.

Proof. This result follows from the existence of Poincaré inequalities on $\partial \Delta$ and from $[\mathrm{HK}]$ theorem 5.7.

\section{Quasi-symmetric homeomorphisms of $\partial \Delta$}

This part is devoted to the proof of the following result (see section 0.2 for the definitions).

3.0 Theorem. Let $\Delta$ and $\Delta^{\prime}$ be two right-angled Fuchsian buildings. Then, any quasi-symmetric homeomorphism $f: \partial \Delta \rightarrow \partial \Delta^{\prime}$ is conformal. 
In section 3.1, we prove a differentiability property of quasi-symmetric homeomorphism with respect to the family of boundaries of apartments (proposition 3.1.1). In section 3.2 a metric version of the classical Rademacher-Stepanov theorem is established (proposition 3.2.1). Theorem 3.0 then follows easily.

Notations. We denote by $\delta$ the combinatorial metric on $\partial \Delta$ with respect to a fixed chamber $c$, by $H$ its Hausdorff dimension, and by $\mathcal{H}$ its Hausdorff measure. The sets $\mathcal{A}, \mathcal{A}_{\xi}$ (for $\xi \in \partial \Delta$ ) and the compact group $K$ have been defined in section 2.2.A, 2.2.B and 2.2. C respectively. Recall that $K$ acts transitively on $\mathcal{A}$. We denote by $d a$ the probability measure on $\mathcal{A}$ induced by the Haar measure of $K$. The stabilizer in $K$ of $\xi$ (where $\xi \in \partial \Delta$ ) acts transitively on $\mathcal{A}_{\xi}$. We denote by $d a_{\xi}$ the probability measure induced on $\mathcal{A}_{\xi}$. The Hausdorff 1-measure defined on some $a \in \mathcal{A}$ will be denoted by $d l$. Given an orientation on the boundary of the chamber $c$, we obtain an orientation on each element of $\mathcal{A}$ which is invariant under retractions centered at $c$. Let $d$ be the common diameter of each element of $\mathcal{A}$. For $a \in \mathcal{A}_{\xi}$, we denote by $\left.a(t), t \in\right]-d, d[$, the unique arc length parameterization of $a$ which is compatible with its orientation and so that $a(0)=\xi$.

\subsection{Differentiability}

Let $(Y, d)$ be an Ahlfors-regular space with the same dimension as $\partial \Delta$ and let $f: \partial \Delta \rightarrow Y$ be a quasi-symmetric homeomorphism. For $\xi \in \partial \Delta$ and $t \in]-d, d[$, set

$$
D_{\xi, t}(a)=\frac{d(f(a(t)), f(\xi))}{|t|}
$$

whenever $a \in \mathcal{A}_{\xi}$.

3.1.1 Proposition. (i) For $\mathcal{H}$-almost every $\xi \in \partial \Delta$, the functions $D_{\xi, t}, t \in$ ]$-d, d\left[\right.$, converge uniformly on $\mathcal{A}_{\xi}$ (when $t$ tends to 0 ) to a constant function whose value denoted by $f^{\prime}(\xi)$ belongs to $(0,+\infty)$.

(ii) If $Y=\partial \Delta^{\prime}$ where $\Delta^{\prime}$ is a right-angled Fuchsian building, then for almost every $\eta \in \partial \Delta^{\prime}$, we have

$$
\left(f^{-1}\right)^{\prime}(\eta)=\frac{1}{f^{\prime}\left(f^{-1}(\eta)\right)} .
$$

The proof is divided into several lemmas.

For $a \in \mathcal{A}$ and $\xi \in a$, set (if this limit exists)

$$
\frac{\partial f}{\partial a}(\xi)=\lim _{t \rightarrow 0} D_{\xi, t}(a) .
$$

If $f(a)$ is a rectifiable curve of $Y$, the next result shows that $\frac{\partial f}{\partial a}(\xi)$ exists for $d l$-almost every $\xi \in a$. 
Lemma 1. Let $(Y, d)$ be a metric space and let $\gamma:[a, b] \rightarrow Y$ be a rectifiable curve. Then, for almost every $x \in[a, b]$, the limit

$$
\lim _{\Delta x \rightarrow 0} \frac{d(\gamma(x+\Delta x), \gamma(x))}{|\Delta x|}
$$

exists and belongs to $(0,+\infty)$.

Proof. Denote by $h(x)$ the length of the sub-curve $\gamma([a, x])$. This function $h$ is continuous and increasing on $[a, b]$ and hence is derivable almost everywhere (see [Fe], 2.9.19).

Let $\gamma_{s}:[0, l(\gamma)] \rightarrow Y$ be the arc length parameterization of $\gamma$. Then, we have $\gamma=\gamma_{s} \circ h$. Maximal intervals with non empty interior on which $h$ is constant are disjoint. Therefore, they are countable. Let $L$ be the complement in $[a, b]$ of their union. It is enough to show that the limit exists for $x \in L$. Whenever $x \in L$, we have

$$
\frac{d(\gamma(x+\Delta x), \gamma(x))}{|\Delta x|}=\frac{d\left(\gamma_{s}(t+\Delta t), \gamma_{s}(t)\right)}{|\Delta t|} \frac{|h(x+\Delta x)-h(x)|}{|\Delta x|}
$$

where $t=h(x)$ and $\Delta t=h(x+\Delta x)-h(x)$. The first term of the right-hand side is less than 1 . Moreover, when $\Delta t$ tends to 0 , it tends to 1 almost everywhere on $[0, l(\gamma)]$ (see $[\mathrm{MM}]$, lemma 9.1). Let $N$ be the set of points of $[0, l(\gamma)]$ where it does not tend to 1 . For almost every $x \in L \backslash h^{-1}(N)$, we have

$$
\lim _{\Delta x \rightarrow 0} \frac{d(\gamma(x+\Delta x), \gamma(x)))}{|\Delta x|}=h^{\prime}(x) .
$$

Since $N$ has zero measure, the Radon-Lebesgue-Nikodym theorem implies that $h^{\prime}$ vanishes almost everywhere on $h^{-1}(N)$. Therefore, equality (1) remains true almost everywhere on $L \cap h^{-1}(N)$, then on $[a, b]$.

Lemma 2. For $\mathcal{H}$-almost every $\xi \in \partial \Delta$, the derivative $\frac{\partial f}{\partial a}(\xi)$ exists da $a_{\xi}$-almost everywhere on $\mathcal{A}_{\xi}$.

Proof. We first show that for $d a$-almost every $a \in \mathcal{A}, f(a)$ is a rectifiable curve in $\partial \Delta^{\prime}$. By proposition 2.3.3, it is enough to prove that, if $\mathcal{F}$ is a Borel set of $\mathcal{A}$ with $\operatorname{Mod}(\mathcal{F})=0$, then $d a(\mathcal{F})=0$. To see this, consider a retraction $\rho$ centered at $c$ onto an apartment $A_{0}$. Denote by $a_{0}$ the boundary of $A_{0}$ and let $\Pi$ be the surjective continuous projection:

$$
\begin{array}{rlc}
\Pi: \mathcal{A} \times a_{0} & \rightarrow & \partial \Delta \\
(a, \zeta) & \mapsto & a \cap \rho^{-1}(\zeta) .
\end{array}
$$

Observe that proposition 2.2.3 (ii) means that

$$
C^{-1} \Pi_{*}(d a \times d l) \leq \mathcal{H} \leq C \Pi_{*}(d a \times d l) .
$$


Therefore, for all $\mathcal{F}$-admissible function $u: \partial \Delta \rightarrow \mathbf{R}$, we have by Hölder inequalities:

$$
\begin{aligned}
\int_{\partial \Delta} u^{H} d \mathcal{H} & \geq C^{-1} \int_{\mathcal{A}}\left(\int_{a} u^{H}(\xi) d l(\xi)\right) d a \\
& \geq C^{-1} \int_{\mathcal{F}}\left(\int_{a} u^{H}(\xi) d l(\xi)\right) d a \\
& \geq C^{-1} L^{1-H} d a(\mathcal{F})
\end{aligned}
$$

( $L$ denotes here the common length of elements of $\mathcal{A}$ ).

By taking the infimum in the previous inequality, we obtain

$$
\operatorname{Mod}(\mathcal{F}) \geq C^{-1} L^{1-H} d a(\mathcal{F}),
$$

which gives the result.

By lemma 1 , this implies that for $d a$-almost every $a \in \mathcal{A}$, the derivative exists $d l$-almost surely on $a$. Consider now the following closed subset of $\partial \Delta \times \mathcal{A}$

$$
T=\left\{(\xi, a) ; \xi \in \partial \Delta, a \in \mathcal{A}_{\xi}\right\} .
$$

Define $\phi: \mathcal{A} \times a_{0} \rightarrow T$ by $\left.\phi(a, \zeta)=(\Pi(a, \zeta), a)\right)$ and note that $\phi$ is a homeomorphism. We will show that, in the coordinate system of $T$, the measure $d a \times d l$ is equal to $m(\xi) \times d a_{\xi}(a)$ where $m=\Pi_{*}(d a \times d l)$, namely for every non negative measurable function $h$ on $T$,

$$
\int_{\partial \Delta}\left(\int_{\mathcal{A}_{\xi}} h(\xi, a) d a_{\xi}(a)\right) d m(\xi)=\int_{\mathcal{A} \times a_{0}}(h \circ \phi) d a \times d l .
$$

By inequalities (1), this will complete the proof of lemma 2. With the notations of 2.2.C, the left-hand side of (2) is equal to $\int_{a_{0}} k(\zeta) d l(\zeta)$ where

$$
k(\zeta)=\int_{\rho^{-1}(\zeta)}\left(\int_{\mathcal{A}_{\xi}} h(\xi, a) d a_{\xi}(a)\right) d \nu_{\zeta}(\xi) .
$$

Since the probability measures $\nu_{\zeta}(\xi) \times d a_{\xi}(a)$ and $d a$ are $K$-invariant and since $\phi(., \zeta)$ is $K$-equivariant, it follows from transitivity that

$$
k(\zeta)=\int_{\mathcal{A}} h \circ \phi(a, \zeta) d a,
$$

and the proof of the lemma is complete.

Lemma 3. For $\mathcal{H}$-almost every $\xi \in \partial \Delta$, the derivative $\frac{\partial f}{\partial a}(\xi)$ is da $a_{\xi}$-almost surely constant on $\mathcal{A}_{\xi}$. 
Proof. Let $\xi$ be a point of $\partial \Delta$ where the derivative $\frac{\partial f}{\partial a}(\xi)$ exists for $d a_{\xi}$-almost every $a \in \mathcal{A}_{\xi}$ (see lemma 2). Let $K_{\xi}$ be the stabilizer in $K$ of $\xi$ and fix $a_{0}$ in $\mathcal{A}_{\xi}$. We consider the measurable function $h$ defined almost everywhere on $K_{\xi}$ by $h(g)=\frac{\partial f}{\partial a}(\xi)$ where $a=g a_{0}$. By transitivity of $K_{\xi}$ on $\mathcal{A}_{\xi}$ and by definition of the probability measure $d a_{\xi}$, it is enough to show that $h$ is right $K_{\xi}$-invariant.

Let $A_{0}$ be the apartment of $\Delta$ whose boundary is $a_{0}$. Let $m$ be a wall of $A_{0}$ which contains an edge of $c$ and so that $c$ and $\xi$ are in the same connected component of $A_{0} \backslash m$. Denote by $\zeta_{-}$and $\zeta_{+}$the endpoints of $m$ of $a_{0}$. Let $L_{-}$ (respectively $L_{+}$) be the stabilizer in $K_{\xi}$ of the sub-arc $S_{-}=\left[\xi, \zeta_{-}\right]$(respectively $\left.S_{+}=\left[\xi, \zeta_{+}\right]\right)$in $a_{0}$.

The function $h$ is invariant under right actions of $L_{+}$and $L_{-}$, since left and right derivatives are equal. We will show that $L_{-}$and $L_{+}$generate $K_{\xi}$, this will complete the proof.

Let $g$ be an element of $K_{\xi}$. By cutting along the tree-wall containing $m$ and along a geodesic ray joining $m \cap c$ to $\xi$, it is easy to construct an element $a_{-}$of $\mathcal{A}_{\xi}$ which contains $S_{-}$and $g S_{+}$. Let $g_{-}$be an element of $L_{-}$so that $g_{-} a_{0}=a_{-}$. Then, $g_{-}^{-1} g a_{0}$ contains $S_{+}$, and therefore $g_{-}^{-1} g \in L_{+}$.

We now prove proposition 3.1.1.

Proof of 3.1.1. We first show part (i). By lemma 3, for almost every $\xi \in \partial \Delta$, the sequence of functions $\left.D_{\xi, t}, t \in\right]-d, d\left[\right.$, tends $d a_{\xi}$-almost everywhere, when $t$ tends to 0 , to a constant function on $\mathcal{A}_{\xi}$. We denote by $f^{\prime}(\xi)$ its value. By corollary 2.3.2, we can assume that $L_{f}(\xi)<\infty$. This last condition shows that

$$
M:=\sup \left\{\frac{d(f(\xi), f(\eta))}{\delta(\xi, \eta)} ; \eta \in \partial \Delta \backslash\{\xi\}\right\}
$$

is finite.

Let $t \in]-d, d\left[\right.$ and let $a_{0} \in \mathcal{A}_{\xi}$. We would like to give an upper bound not depending on $a_{0}$ of the following quantity

$$
\alpha:=\left|D_{\xi, t}\left(a_{0}\right)-f^{\prime}(\xi)\right| .
$$

For this, consider $\zeta=a_{0}(t)$. Set $T=d(f(\xi), f(\zeta))$ and let $v=\frac{\alpha}{2 M}$. Then, for every $y \in B(f(\zeta), v T)$

$$
\begin{aligned}
\left|\frac{d(f(\xi), y)}{|t|}-f^{\prime}(\xi)\right| & \geq\left|\frac{d(f(\xi), f(\zeta))}{|t|}-f^{\prime}(\xi)\right|-\left|\frac{d(f(\xi), y)-d(f(\xi), f(\zeta)))}{|t|}\right| \\
& \geq \alpha-\frac{d(f(\zeta), y)}{|t|} \\
& \geq \alpha-\frac{v T}{|t|}=\alpha-\frac{\alpha T}{2 M|t|}
\end{aligned}
$$


Using the definition of $M$, we obtain for every $y \in B(f(\zeta), v T)$,

$$
\left|\frac{d(f(\xi), y)}{|t|}-f^{\prime}(\xi)\right| \geq \frac{\alpha}{2}
$$

Let $\phi$ be the homeomorphism of quasi-symmetry of $f$ (see 0.2 ). Set $u=\min \left\{\phi^{-1}(v), 1\right\}$. Then, we have

$$
f(B(\zeta, u t)) \subset B(f(\zeta), v T) .
$$

Let $\mathcal{E}_{a_{0}, t, u}$ be the following closed subset of $\mathcal{A}_{\xi}$

$$
\mathcal{E}_{a_{0}, t, u}=\left\{a \in \mathcal{A}_{\xi} ; a(t) \in B(\zeta, u t)\right\},
$$

(as above $\zeta$ denotes $a_{0}(t)$ ). Applying proposition 2.2.4 to $\xi=\eta$, there exists a constant $C \geq 1$, depending only on $\Delta$, so that

$$
d a_{\xi}\left(\mathcal{E}_{a_{0}, t, u}\right) \geq C^{-1} u^{H-1} .
$$

Indeed, with the same notations as in 2.2.4, the measure induced by $d a_{\xi}$ on $F_{\xi}^{\zeta}$ is equal, by transitivity of $\operatorname{Stab}_{K}(\xi)$, to

$$
\left.\frac{1}{\nu_{\zeta}\left(F_{\xi}^{\zeta}\right)} \nu_{\zeta}\right|_{F_{\xi}^{\zeta}}
$$

Relations (2) and (1) imply that for $a \in \mathcal{E}_{a_{0}, t, u}$,

$$
\left|D_{\xi, t}(a)-f^{\prime}(\xi)\right| \geq \frac{\alpha}{2}
$$

By (3), it follows

$$
\begin{aligned}
\int_{\mathcal{A}_{\xi}}\left|D_{\xi, t}(a)-f^{\prime}(\xi)\right| d a_{\xi} & \geq \frac{\alpha}{2} d a_{\xi}\left(\mathcal{E}_{a_{0}, t, u}\right) \\
& \geq C^{-1} \frac{\alpha}{2}\left(\min \left(\phi^{-1}\left(\frac{\alpha}{2 M}\right), 1\right)\right)^{H-1} .
\end{aligned}
$$

By the Lebesgue dominated convergence theorem, the integral above tends to 0 when $t$ tends to 0 . Therefore, $D_{\xi, t}$ tends uniformly on $\mathcal{A}_{\xi}$ to $f^{\prime}(\xi)$. This completes the proof of (i).

We now show (ii). Let $\eta$ be a point of $\partial \Delta^{\prime}$ such that (i) of proposition 3.1.1 is satisfied for $\eta$ and for $\xi=f^{-1}(\eta)$, and such that proposition 2.2.2 is satisfied for $\xi$ (see 2.3.1 (ii)). Let $a^{\prime}$ be an element of $\mathcal{A}_{\eta}$ and denote by $\gamma(T)$ the curve $f^{-1}\left(a^{\prime}(T)\right)$. By 2.2.2, there exists a sequence $\left\{T_{n}\right\}$ of real numbers tending to 0 and a sequence $\left\{a_{n}\right\}$ of elements of $\mathcal{A}_{\xi}$ so that $\gamma\left(T_{n}\right)$ belongs to $a_{n} \backslash\{\xi\}$. Set $t_{n}=\delta\left(\xi, \gamma\left(T_{n}\right)\right)$. Then, $\left(f^{-1}\right)^{\prime}(\eta)=\lim _{n \rightarrow+\infty} \frac{t_{n}}{\left|T_{n}\right|}$ and $\frac{t_{n}}{\left|T_{n}\right|}=\left(D_{\xi, t_{n}}\left(a_{n}\right)\right)^{-1}$. We conclude using the uniform convergence. 


\subsection{A Rademacher-Stepanov type theorem}

By adapting Rademacher-Stepanov's arguments (see [V2], theorem 29.1), we prove the following result.

3.2.1 Proposition. Under the same hypothesis as in proposition 3.1.1, for almost every $\xi \in \partial \Delta$, we have $L_{f}(\xi)=f^{\prime}(\xi)$.

Before going through the proof of this proposition, let us explain how to deduce the proof of theorem 3.0 from 3.2.1 and 3.1.1.

Proof of theorem 3.0. By proposition 2.3 .1 (i), $\partial \Delta$ and $\partial \Delta^{\prime}$ have the same Hausdorff dimension (recall that the inverse of a quasi-symmetric homeomorphism is quasi-symmetric). Moreover, by propositions 3.2.1 and 3.1.1 (ii), for almost every $\xi \in \partial \Delta$, we have $L_{f}(\xi)=f^{\prime}(\xi)$ and

$$
L_{f^{-1}}(f(\xi))=\left(f^{-1}\right)^{\prime}(f(\xi))=\frac{1}{f^{\prime}(\xi)} .
$$

But in full generality, one has

$$
L_{f^{-1}}(f(\xi))=\frac{1}{l_{f}(\xi)} .
$$

Hence, $L_{f}(\xi)$ is almost everywhere equal to $l_{f}(\xi)$.

We now give the proof of proposition 3.2.1.

Proof of 3.2.1. For $i \in \mathbf{N}^{*}$, set

$$
V_{i}=\{\xi \in \partial \Delta ; \forall \eta \in B(\xi, 1 / i), d(f(\xi), f(\eta)) \leq i \delta(\xi, \eta)\} .
$$

Since $L_{f}(\xi)<\infty$ almost everywhere, we have $\mathcal{H}\left(\partial \Delta \backslash \cup_{i} V_{i}\right)=0$ (see corollary 2.3.2).

Let $\varepsilon>0$ and consider $i_{0} \in \mathbf{N}$ such that $\mathcal{H}\left(\partial \Delta \backslash V_{i_{0}}\right) \leq \varepsilon / 2$. For $r>0$, set

$$
h_{r}(\xi)=\sup \left\{\left|D_{\xi, t}(a)-f^{\prime}(\xi)\right| ; a \in \mathcal{A}_{\xi},-r \leq t \leq r\right\} .
$$

Proposition 3.1.1 shows that $h_{r}$ tends almost everywhere to 0 when $r$ tends to 0 . By Egoroff theorem, there exists a compact set $F$ in $\partial \Delta$ so that $\mathcal{H}(\partial \Delta \backslash F) \leq \varepsilon / 2$ and so that $h_{r}$ converges uniformly to 0 on $F$ when $r$ tends to 0 . Note that $f^{\prime}$ is continuous on $F$.

Let $E=F \cap V_{i_{0}}$ and let $G$ be the set of density points of $E$. Then, we have $\mathcal{H}(\partial \Delta \backslash E) \leq \varepsilon$ and $\mathcal{H}(E \backslash G)=0$. Hence, it is enough to show that $L_{f}(\xi)=f^{\prime}(\xi)$ for every $\xi \in G$. 
To this issue, fix $\xi$ in $G$ and define the following functions for $r \leq 1 / i_{0}$ :

$$
\begin{gathered}
\varepsilon_{1}(r)=\frac{\mathcal{H}(B(\xi, r) \backslash E)}{\mathcal{H}(B(\xi, r))} ; \\
\varepsilon_{2}(r)=\sup \left\{h_{r}(\eta) ; \eta \in F\right\} ; \\
\varepsilon_{3}(r)=\sup \left\{\left|f^{\prime}(\xi)-f^{\prime}(\eta)\right| ; \eta, \xi \in F, \delta(\xi, \eta) \leq r\right\} .
\end{gathered}
$$

These functions converge to 0 when $r$ tends to 0 . Let $\xi^{\prime}$ be a point of $B(\xi, r)$. By proposition 2.2.1, there exists a geodesic segment $\left[\xi, \xi^{\prime}\right]$ in $\partial \Delta$ which is the union of at most 4 geodesic segments $\left[\xi, \xi_{1}\right],\left[\xi_{1}, \xi_{2}\right],\left[\xi_{2}, \xi_{3}\right],\left[\xi_{3}, \xi^{\prime}\right]$ contained in elements of $\mathcal{A}$. We set $\xi_{4}=\xi^{\prime}$.

For $\theta \in \partial \Delta$ and $\zeta \in \mathcal{A}_{\theta}$, define

$$
\mathcal{E}_{\theta}(\zeta, t)=\bigcup_{a \in \mathcal{A}_{\theta}} a \cap B(\zeta, t)
$$

whenever $t>0$. Propositions 2.2.3 (ii) and 2.2.4 (ii) (applied with $\xi=\eta=\theta$ ), and the Ahlfors-regularity of $\mathcal{H}$ imply that there exists a constant $C \geq 1$ so that

$$
\mathcal{H}\left(\mathcal{E}_{\theta}(\zeta, t)\right) \geq C^{-1} \mathcal{H}(B(\zeta, t)) .
$$

Let $\zeta \in B(\xi, r) \cap \mathcal{A}_{\theta}$ and let $t \leq r$. Then we obtain

$$
\begin{aligned}
\frac{\mathcal{H}\left(\mathcal{E}_{\theta}(\zeta, t) \backslash E\right)}{\mathcal{H}\left(\mathcal{E}_{\theta}(\zeta, t)\right)} & \leq C \frac{\mathcal{H}(B(\zeta, t) \backslash E)}{\mathcal{H}(B(\zeta, t))} \\
& \leq C^{\prime} \frac{\mathcal{H}(B(\xi, 2 r) \backslash E)}{\mathcal{H}(B(\xi, 2 r))} \frac{(2 r)^{H}}{t^{H}} \\
& \leq C^{\prime} \varepsilon_{1}(2 r) \frac{(2 r)^{H}}{t^{H}}
\end{aligned}
$$

where $C^{\prime}$ is a constant depending only on $C$ and on the regularity constant of $\mathcal{H}$. We set

$$
t(r)=4 r\left(C^{\prime} \varepsilon_{1}(2 r)\right)^{1 / H} .
$$

For $t=t(r)$, the right-hand side member of the last inequality above is strictly less than 1 . Therefore, $\mathcal{E}_{\theta}(\zeta, t(r)) \cap E$ is not empty. have

Hence, for $i \in\{1,2,3\}$, let $\eta_{i}$ be an element of $\mathcal{E}_{\xi_{i+1}}\left(\xi_{i}, t(r)\right) \cap E$. Then, we

$$
\begin{aligned}
d\left(f(\xi), f\left(\xi^{\prime}\right)\right) & \leq d\left(f(\xi), f\left(\xi_{1}\right)\right)+\sum_{i=1}^{3} d\left(f\left(\eta_{i}\right), f\left(\xi_{i+1}\right)\right)+\sum_{i=1}^{3} d\left(f\left(\xi_{i}\right), f\left(\eta_{i}\right)\right) \\
& \leq f^{\prime}(\xi) \delta\left(\xi, \xi_{1}\right)+\sum_{i=1}^{3} f^{\prime}\left(\eta_{i}\right) \delta\left(\eta_{i}, \xi_{i+1}\right)+4 r \varepsilon_{2}(t)+3 i_{0} t(r) \\
& \leq f^{\prime}(\xi) r+3 r \varepsilon_{3}(r)+3\left(f^{\prime}(\xi)+\varepsilon_{3}(r)\right) t(r)+4 r \varepsilon_{2}(t)+3 i_{0} t(r) .
\end{aligned}
$$


Since $t(r) / r$ converges to 0 when $r$ tends to 0 , we obtain $L_{f}(\xi) \leq f^{\prime}(\xi)$, and then the equality.

Remark. Let $f$ be as in proposition 3.1.1. From propositions 2.2.2, 3.1.1 and 3.2.1, and by similar arguments as in the proof of proposition 4.1 .1 of the next section, one can show that $f$ contracts moduli. Namely, for all family $\mathcal{F}$ of curves of $\partial \Delta$, one has

$$
\operatorname{Mod}(f(\mathcal{F})) \leq \operatorname{Mod}(\mathcal{F})
$$

\section{A Liouville type theorem for $\partial \Delta$}

If $S$ and $S^{\prime}$ are non-compact symmetric spaces of rank one (and different from $H_{\mathbf{R}}^{2}$ ), a classical theorem due to Liouville and generalized by Mostow (see [Mo]) shows that any conformal homeomorphism from the boundary of $S$ to the boundary of $S^{\prime}$ is induced by an isometry from $S$ to $S^{\prime}$. The main goal of this part is to give an analogous statement in the setting of right-angled Fuchsian buildings.

4.0 Theorem. Let $\Delta$ and $\Delta^{\prime}$ be two right-angled Fuchsian buildings and let $f: \partial \Delta \rightarrow \partial \Delta^{\prime}$ be a conformal homeomorphism. Then, $f$ is the extension to the boundaries of an isometry from $\Delta$ to $\Delta^{\prime}$.

Our proof works in the setting of symmetric spaces too. According to 0.2, theorem 4.0 completes the proof of the main theorem.

This part is organized as follows. In 4.1, we give a precise estimate on the size of the conformal group of a Loewner space (proposition 4.1.3); it is basically due to J. Ferrand $[\mathrm{F}]$. In section 4.2 , we combine it with Sullivan's criterion given in 2.1.9 to prove theorem 4.0.

\subsection{The conformal group of a Loewner space}

We start with an observation about conformal homeomorphisms. The notion of modulus has been defined in section 2.3.B.

\section{A. Preservation of moduli.}

4.1.1 Proposition. Let $X$ and $Y$ be two Ahlfors regular metric spaces with the same dimension $H$ and let $f: X \rightarrow Y$ be a conformal homeomorphism. Then, $f$ is absolutely continuous. Moreover, if $H>1$, then $f$ is absolutely continuous along almost every rectifiable curves and $f$ preserves moduli:

$$
\operatorname{Mod}(f(\mathcal{F}))=\operatorname{Mod}(\mathcal{F})
$$

for every family $\mathcal{F}$ of curves of $X$. 
The proof will use the following lemma.

Lemma. Let $X$ and $Y$ be two metric spaces. We assume that the Hausdorff measures of dimension $H$ of $X$ and $Y$ are locally bounded. Let $f: X \rightarrow Y$ be a homeomorphism and let $\mu_{f}$ be the derivative of $f^{*} \mathcal{H}_{Y}$ with respect to $\mathcal{H}_{X}$. Then, for $\mathcal{H}_{X}$-almost every $x \in X$, we have

$$
\left(l_{f}(x)\right)^{H} \leq \mu_{f}(x) \leq\left(L_{f}(x)\right)^{H} .
$$

Proof. Since this result is local, we can assume that $\mathcal{H}_{X}$ and $\mathcal{H}_{Y}$ are bounded on $X$ and $Y$ respectively. For $t>0$ and $x \in X$, set

$$
L_{f}^{t}(x)=\sup _{r \leq t} \frac{L_{f}(x, r)}{r} .
$$

We will show that $\mu_{f} \leq\left(L_{f}^{t}\right)^{H}$ almost everywhere, then the right-hand side inequality will follow by taking the limit when $t$ tends to 0 . Consider the set

$$
E=\left\{x \in X ; L_{f}^{t}(x)<+\infty\right\} .
$$

Let $B$ be a Borel set of $E$ and let $\varepsilon>0$. Since $\mathcal{H}_{X}(B)$ is finite, there exists a countable partition $\left\{V_{i}\right\}$ of $B$ by Borel sets and there exist real numbers $c_{i}>0$ so that, for $x \in B$,

$$
\left(L_{f}^{t}(x)\right)^{H} \leq \sum_{i} c_{i} \mathbf{1}_{V_{i}}(x)
$$

with

$$
\sum_{i} c_{i} \mathcal{H}_{X}\left(V_{i}\right) \leq \int_{B}\left(L_{f}^{t}\right)^{H} d \mathcal{H}_{X}+\varepsilon
$$

Since the restriction of $f$ to $V_{i}$ is locally $c_{i}^{1 / H}$-Lipschitz, we have $\mathcal{H}_{Y}\left(f\left(V_{i}\right)\right) \leq$ $c_{i} \mathcal{H}_{X}\left(V_{i}\right)$. It follows

$$
\begin{aligned}
\int_{B} \mu_{f} d \mathcal{H}_{X} & \leq \mathcal{H}_{Y}(f(B))=\sum_{i} \mathcal{H}_{Y}\left(f\left(V_{i}\right)\right) \\
& \leq \sum_{i} c_{i} \mathcal{H}_{X}\left(V_{i}\right) \\
& \leq \int_{B}\left(L_{f}^{t}\right)^{H} d \mathcal{H}_{X}+\varepsilon
\end{aligned}
$$

The other inequality can be proved by similar methods.

We now prove proposition 4.1.1. 
Proof of 4.1.1. The previous lemma implies that

$$
\left(l_{f}\right)^{H}=\mu_{f}=\left(L_{f}\right)^{H} \text { a.e.. }
$$

Hence, $\mu_{f}$ and $\mu_{f-1}$ (by changing the role of $f$ and $f^{-1}$ ) belong almost everywhere to $(0,+\infty)$. Therefore, the Lebesgue-Radon-Nikodym theorem shows that $f$ and $f^{-1}$ are absolutely continuous. The absolute continuity along almost every rectifiable curves follows henceforth from $[\mathrm{T}]$ corollary 1.8 (see also $[\mathrm{Pa}], 4.6$ ).

We now show that $f$ preserves moduli. For this, let $\mathcal{F}$ be a family of curves of $X$ and denote by $\mathcal{F}^{\prime}$ the sub-family of $\mathcal{F}$ of curves along which $f$ is absolutely continuous. We have

$$
\operatorname{Mod}(\mathcal{F})=\operatorname{Mod}\left(\mathcal{F}^{\prime}\right)
$$

For an $f(\mathcal{F})$-admissible function $v$ of $Y$, let $u$ be the following function of $X$

$$
u(x)=v(f(x)) L_{f}(x)
$$

Since $f$ is absolutely continuous along curves of $\mathcal{F}^{\prime}$, standard arguments show that $u$ is $\mathcal{F}^{\prime}$-admissible. Equality (1) and the absolute continuity of $f$ imply

$$
\begin{aligned}
\int_{X} u^{H} d \mathcal{H}_{X} & =\int_{X}(v \circ f(x))^{H} \mu_{f}(x) d \mathcal{H}_{X} \\
& =\int_{Y} v^{H} d \mathcal{H}_{Y} .
\end{aligned}
$$

This last equality and $(2)$ imply $\operatorname{Mod}(\mathcal{F}) \leq \operatorname{Mod}(f(\mathcal{F}))$. By changing the role of $f$ and $f^{-1}$, we obtain the desired equality.

B. J. Ferrand's cross-ratio. Let $X$ be an Ahlfors-regular Loewner space with dimension $H>1$. We assume that for every 4 -tuples $\left(x_{1}, y_{1}, x_{2}, y_{2}\right)$ of distinct points of $X$, we can find disjoint continua $C_{1}$ and $C_{2}$ so that $x_{1}, y_{1} \in C_{1}$ and $x_{2}, y_{2} \in C_{2}$. Following J. Ferrand (see $[\mathrm{F}]$ ), we define their cross-ratio by

$$
b\left(x_{1}, y_{1}, x_{2}, y_{2}\right)=\inf \operatorname{Mod}\left(C_{1}, C_{2}\right)
$$

where the infimum is taken over all disjoint continua $C_{1}, C_{2}$ such that $x_{1}, y_{1} \in C_{1}$ and $x_{2}, y_{2} \in C_{2}$. The two propositions below have been proved by J. Ferrand in the setting of Riemannian manifolds. Here, they follow from the Loewner property.

4.1.2. Proposition. The function $b$ is continuous on the set of 4-tuples of pairwise distinct points of $X$. It takes its values in $(0,+\infty)$. It admits a continuous extension to the set of 4-tuples with at least 3 distinct coordinates, by

$$
b\left(x_{1}, y_{1}, x_{2}, y_{2}\right)=0 \text { if } x_{1}=y_{1} \text { or } x_{2}=y_{2},
$$




$$
b\left(x_{1}, y_{1}, x_{2}, y_{2}\right)=\infty \text { if }\left\{x_{1}, y_{1}\right\} \cap\left\{x_{2}, y_{2}\right\} \neq \varnothing .
$$

Proof. On the set of 4-tuples of pairwise distinct points, $b$ is positive by the Loewner property. To prove the finiteness of $b$, we cover two disjoint continua $C_{1}$ and $C_{2}$ by balls with small radius in comparison with $\operatorname{dist}\left(C_{1}, C_{2}\right)$. Then, we apply properties (a) and (b) of moduli recalled in subsection 2.3.B.

Since $X$ is a Loewner space, there exists a constant $C>1$ so that, for every ball $B(x, r)$ and every point $z \in B(x, r)$, there exists a path in $B(x, C r)$ joining $x$ to $z$ (see $[\mathrm{HK}]$, theorem 3.13). Moreover, the Loewner property gives us a lower bound of the distance between continua $C_{1}$ and $C_{2}$ which almost realize the infimum. Combined with properties (a) and (b) of modulus, we obtain the continuity.

Similar methods show the continuous extension.

Assume now that $X$ is compact. We denote by $C(X)$ and $Q S(X)$ the groups of conformal homeomorphisms and of quasi-symmetric homeomorphisms of $X$. Let $M(X)$ be the group of homeomorphisms of $X$ preserving J. Ferrand's cross-ratio. We endow this group with the uniform distance.

4.1.3. Proposition. (i) We have $C(X) \subset M(X) \subset Q S(X)$.

(ii) The group $M(X)$ acts properly on the set of triples of pairwise distinct points of $X$.

Proof. The first inclusion of (i) follows from proposition 4.1.1. The second inclusion follows from a classical argument using Loewner property, which is due to Loewner and Gehring (see for example [HK], proof of theorem 4.7).

Part (ii) is due to J. Ferrand (see $[\mathrm{F}]$, theorem A(a)), and the proof uses only the continuity properties of the cross-ratio stated in 4.1.2 and Ascoli theorem.

4.1.4 Remark. Here is a straightforward generalization of proposition 4.1.3. Let $X$ be a metric space as in 4.1 .3 and denote by $H(X)$ the group of homeomorphisms equipped with the uniform distance. Let $G$ be a sub-group of $H(X)$. We assume that there exists $K \geq 1$ such that, for every family $\mathcal{F}$ of curves of $X$ and for every element $g \in G$, we have

$$
K^{-1} \operatorname{Mod}(\mathcal{F}) \leq \operatorname{Mod}(g(\mathcal{F})) \leq K \operatorname{Mod}(\mathcal{F})
$$

The same arguments show that $\bar{G}$ (namely the closure of $G$ in $H(X)$ ) is contained in $Q S(X)$ and acts properly on the set of triples of pairwise distinct points of $X$.

\subsection{Proof of theorem 4.0}

Denote by $M$ and $M^{\prime}$ the groups $M(\partial \Delta)$ and $M\left(\partial \Delta^{\prime}\right)$ respectively. We first show that the measure $\mu$ introduced in 2.1.8 is invariant under the diagonal action of $M$ on $\partial^{2} \Delta$. Let $\Gamma$ be a co-compact lattice of $\operatorname{Isom}(\Delta)$. Then, $\Gamma$ is contained in 
$M$ and its action on the set of triples of pairwise distinct points of $\partial \Delta$ is properly discontinuous and co-compact (see $[\mathrm{G}]$ ). Hence, by proposition 4.1.3 (ii), the group $\Gamma$ is a co-compact lattice of $M$. In particular, the Haar measure of $M$ is biinvariant. Let $m$ be the measure on $\Gamma \backslash M$ that we obtain by restricting the Haar measure of $M$ to a fundamental domain of $\Gamma$ in $M$. Note that $m$ is finite and is invariant under the right-action of $M$. We define a new measure $\nu$ on $\partial^{2} \Delta$ by

$$
\nu(B)=\int_{\Gamma \backslash M} \mu(s(\bar{g}) B) d m(\bar{g})
$$

whenever $B$ is a Borel set of $\partial^{2} \Delta$ and $s$ is a measurable section from $\Gamma \backslash M$ into $M$. Since $\mu$ is $\Gamma$-invariant and since $m$ is invariant under the right action of $M$, the measure $\nu$ is $M$-invariant. Moreover, this measure is absolutely continuous with respect to $\mu$. Indeed, $M$ is contained in $Q S(\partial \Delta)$ (see proposition 4.1.3 (ii)) and quasi-symmetric homeomorphisms are absolutely continuous by 2.3 .1 (ii).

Because $\mu$ is $\Gamma$-ergodic (see 2.1.9 (ii)), we obtain that $\mu$ is equal to $C \nu$ where $C$ is a constant. So, $\mu$ is $M$-invariant.

Consider now a conformal homeomorphism $f: \partial \Delta \rightarrow \partial \Delta^{\prime}$. Then, $f$ preserves J. Ferrand's cross-ratio and therefore conjugates the groups $M$ and $M^{\prime}$. Hence, the measure $(f \times f)_{*} \mu$ is $M^{\prime}$-invariant on $\partial^{2} \Delta^{\prime}$. This measure belongs to the class of $\mu^{\prime}$ (this follows from the absolute continuity of $f$ ). By ergodicity, it is equal to $C \mu^{\prime}$ where $C$ is a constant. Using Sullivan's criterion (proposition 2.1.9 (i)), we can complete the proof.

\subsection{General Fuchsian buildings}

In this section, we collect arguments of this paper that can be extended to general Fuchsian buildings (see [B2] chapter 1 for their definition).

Let $\Delta$ be a Fuchsian building. In section 2.B of [B2], are constructed a Gromov product, a combinatorial metric on $\partial \Delta$, and a probability measure on $\mathcal{A}$. Using them, it is easy to generalize the results of subsection 2.2.C. It follows that $\partial \Delta$ admits $(1,1)$ Poincaré inequalities and satisfies the results of section 2.3 , in particular, $\partial \Delta$ is a Loewner space. If we assume in addition that $\operatorname{Isom}(\Delta)$ acts transitively on $\mathcal{A}$ (see [L] for examples of such buildings), proposition 3.1.1 (i) is still true.

However, we do not know if propositions 2.2.1 and 2.2.2 remain true. They have been used to show propositions 3.1.1 (ii) and 3.2.1 which imply theorem 3.0.

The proof of theorem 4.0 follows from the Loewner property of $\partial \Delta$ and from Sullivan's criterion (proposition 2.1.9). We conjecture that this last result is still satisfied by all Fuchsian buildings. 


\section{Acknowledgments}

We would like to thank J. Heinonen and P. Koskela whose paper $[\mathrm{HK}]$ motivated our work. We wish to thank J. Cheeger, F. Haglund, P. Pansu, F. Paulin for their interest and useful discussions. A large part of this work has been done during a stay of the two authors at the University of Bretagne-Sud. We thank its members, and especially R. Leplaideur, for their hospitality. Many thanks to B. Rémy for his help during the final draft of the paper, and to B. Pinçon for the figures made with xfig. The second author would like to dedicate this paper to the memory of his grandfather who died during the writing of this article.

\section{References}

[A] R.D. Anderson, A Characterization of universal curve and a proof of its homogeneity, Annals of Mathematics 67 (1958), 313-324.

[Ba] W. Ballmann, Singular spaces of non-positive curvature, in «Sur les groupes hyperboliques, d'après Mikhael Gromov 》(E. Ghys, P. De La Harpe, eds.), Progress in Mathematics 83 Birkhäuser (1990), 189-202.

[Be] N. Benakli, Polyèdres hyperboliques, passage du local au global, Thèse, Université ParisSud, 1992.

[B1] M. Bourdon, Immeubles hyperboliques, dimension conforme et rigidité de Mostow, Geometric And Functional Analysis 7 (1997), 245-268.

[B2] M. Bourdon, Sur les immeubles fuchsiens et leur type de quasiisométrie, Ergodic Theory and Dynamical Systems 20 (2000), 343-364.

[B3] M. Bourdon, Sur la dimension de Hausdorff de l'ensemble limite d'une famille de sousgroupes convexes cocompacts, Compte Rendus de l'Académie des Sciences de Paris $\mathbf{3 2 5}$ (1997), 1097-1100.

[BP] M. Bourdon, H. Pajot, Poincaré inequalities and quasiconformal structure on the boundary of some hyperbolic buildings, Proceedings of the American Mathematical Society 127 (1999), 2315-2324.

[Br] K.J. Brown, Buildings, Springer-Verlag, 1989.

[CDP] M. Coornaert, T. Delzant, A. Papadopoulos, Géométrie et théorie des groupes, les groupes hyperboliques de M. Gromov, Lecture Notes in Mathematics 1441, SpringerVerlag, 1991.

[EF] A. Eskin, B. Farb, Quasi-flats and rigidity in higher rank symmetric spaces, Journal of the American Mathematical Society 10 (1997), 653-692.

[Fe] H. Federer, Geometric measure theory, Springer-Verlag 1969.

$[\mathrm{F}]$ J. Ferrand, Convergence and degeneracy of quasiconformal maps of Riemannian manifolds, Journal d'Analyse Mathématique 69 (1996), 1-24.

[GP] D. Gaboriau, F. Paulin, Sur les immeubles hyperboliques, Prépublication 98-18, Université d'Orsay, 1998.

[GH] E. Ghys, P. De La Harpe, Sur les groupes hyperboliques, d'après Mikhael Gromov, Progress in Mathematics 83, Birkhäuser, 1990.

[G] M. Gromov, Hyperbolic groups, in «Essays in group theory» (S. M. Gersten, editor), Springer-Verlag (1987), 75-263.

[Hae] A. Haefliger, Complexes of groups and orbiedra, in «Group theory from geometrical viewpoint》(E. Ghys, A. Haefliger, A. Verjovsky, Eds.), World Scientific, Singapore (1991), 504-540.

[H] F. Haglund, Réseaux de Coxeter-Davis et commensurateurs, Annales de l'Institut Fouri- 
er, Grenoble 48 (1998), 649-666.

[HP] F. Haglund, F. Paulin, Simplicité des groupes d'automorphismes d'espaces à courbure négative, Geometry and topology monographs 1 (1998): The Epstein Birthday Schrift (I. Rivin, C. Rourke, C. Series, Eds), 181-248.

[Ham] U. Hamenstädt, Cocycles, Hausdorff measures and cross-ratios, Ergodic Theory and Dynamical Systems 17 (1997), 1061-1081.

[HK] J. Heinonen, P. Koskela, Quasiconformal maps in metric spaces with controlled geometry, Acta Mathematica 181 (1998), 1-61.

[KK] M. Kapovich, B. Kleiner, Hyperbolic groups with low dimensional boundary, Preprint Utah, 1999.

[KL1] B. Kleiner, B. Leeb, Rigidity of quasi-isometries for symmetric spaces and Euclidean buildings, Compte Rendus de l'Académie des Sciences de Paris, Série I 324 (1997), 639-643.

[KL2] B. Kleiner, B. Leeb, Rigidity of quasi-isometries for symmetric spaces and Euclidean buildings, Publications Mathématiques de l'Institut des Hautes Etudes Scientifiques $\mathbf{8 6}$ (1998), 115-197.

[L] E. Lebeau, Rigidité et flexibilité de complexes polyédraux à courbure négative, Thèse ENS Lyon, 1999.

[Led] F. Ledrappier, Structures au bord des variétés à courbure négative, Séminaire de Théorie Spectrale, Grenoble (1994-1995), 97-122.

$[\mathrm{MM}]$ G.A. Margulis, G.D. Mostow, The differential of a quasi-conformal mapping of a CarnotCarathéodory space, Geometric And Functional Analysis 5 (1995), 402-433.

[Ma] B. Maskit, Kleinian groups, Springer-Verlag, 1987.

[Mat] P. Mattila, Geometry of sets and measures in Euclidean spaces, Cambridge Studies in Advanced Mathematics, 44, Cambridge University Press, 1995.

[Mo] G.D. Mostow, Strong rigidity of locally symmetric spaces, Annals of Mathematical Studies 78, Princeton University Press, 1973.

$[\mathrm{Pa}]$ P. Pansu, Métriques de Carnot-Carathéodory et quasiisométries des espaces symétriques de rang un, Annals of Mathematics 129 (1989), 1-60.

[R] B. Rémy, Construction de réseaux en théorie de Kac-Moody, Comptes Rendus de l'Académie des Sciences de Paris 329 (1999), 475-478.

[Ro] M. Ronan, Lectures on buildings, Academic Press, 1988.

[Sc] R. Schwartz, The quasi-isometry classification of rank one lattices, Publications Mathématiques de l'Institut des Hautes Etudes Scientifiques 82 (1995), 133-168.

[S] D. Sullivan, Discrete conformal groups and measurable dynamics, Bulletin (new series) of the American Mathematical Society 6 (1982), 57-73.

[TK] P. Tukia, J. Väisälä, Quasi-symmetric embeddings of metric spaces, Annales Academiae Scientiarum Fennicae (mathematica) 5 (1980), 97-114.

[T] J. Tyson, Quasiconformality and quasisymmetry in metric measure spaces, Annales Academiae Scientiarum Fennicae (mathematica) 23 (1998), 525-548.

[V1] J. Väisälä, Quasiconformal maps of cylindral domains, Acta Mathematica 162 (1989), 201-225.

[V2] J. Väisälä, Lectures on n-dimensional quasiconformal mappings, Lecture Notes in Mathematics 229, Springer-Verlag, 1971. 
Marc Bourdon

Département de Mathématiques

Université de Lille 1

F-59655 Villeneuve d' Ascq Cedex

France

e-mail: bourdon@agat.univ-lille1.fr

\section{Hervé Pajot}

Département de Mathématiques

Université de Cergy-Pontoise

2 avenue Adolphe Chauvin

BP 222, Pontoise

F-95302 Cergy-Pontoise Cédex

France

e-mail: pajot@math.pst.u-cergy.fr

(Received: February 2, 2000) 